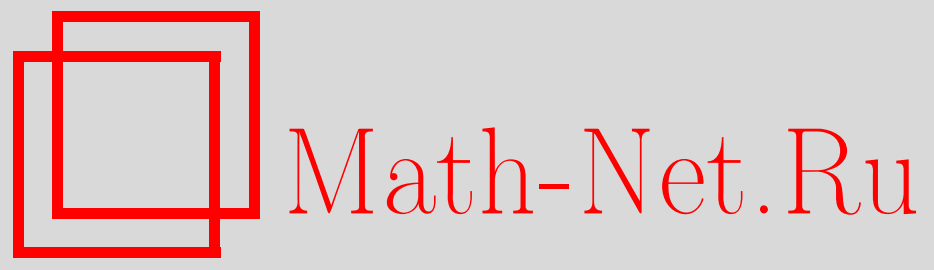

В. И. Богачев, М. Рёкнер, С. В. Шапошников, Оценки плотностей стационарных распределений и переходных вероятностей диффузионных процессов, Теория вероятн. и ее примен., 2007, том 52, выпуск 2, 240270

DOI: https://doi.org/10.4213/tvp172

Использование Общероссийского математического портала Math-Net.Ru подразумевает, что вы прочитали и согласны с пользовательским соглашением

http://www.mathnet.ru/rus/agreement

Параметры загрузки:

IP : 3.91 .87 .62

26 апреля 2023 г., 09:39:50

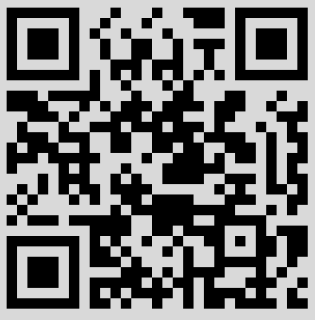




\section{ОЦЕНКИ ПЛОТНОСТЕЙ СТАЦИОНАРНЫХ РАСПРЕДЕЛЕНИЙ И ПЕРЕХОДНЫХ ВЕРОЯТНОСТЕЙ ДИФФУЗИОННЫХ ПРОЦЕССОВ ${ }^{1)}$}

Получены нижние оценки решений эллиптических и параболических уравнений второго порядка на всем пространстве. Метод основан на изучении зависимости постоянной в неравенстве Харнака от коэффициентов уравнения. В качестве применения получены нижние оценки плотностей стационарных распределений и переходных вероятностей диффузионных процессов с неограниченными коэффициентами сноса.

Ключевые слова и фразы: неравенство Харнака, переходная вероятность, стационарное распределение, нижние оценки решений параболических уравнений.

1. Введение. Цель этой работы состоит в том, чтобы получить оценки снизу для плотностей стационарных распределений и переходных вероятностей диффузионных процессов с неограниченными (возможно, быстро растущими) коэффициентами сноса. Для этого будут получены нижние оценки плотностей решений эллиптических и параболических уравнений вида

$$
\mathscr{L}^{*} \mu=0
$$

для борелевских мер $\mu$ на $\mathbf{R}^{d}$ или на $\mathbf{R}^{d} \times(0,1)$ соответственно. Здесь $\mathscr{L}$ - эллиптический или параболический оператор второго порядка вида

$$
\mathscr{L} \varphi(x):=\partial_{x_{i}}\left(a^{i j}(x) \partial_{x_{j}} \varphi(x)\right)+b^{i}(x) \partial_{x_{i}} \varphi(x)
$$

* Московский государственный университет им. М.В. Ломоносова, механикоматематический факультет, Ленинские горы, 119992 Москва, Россия; е-mail: sfb1@mathematic.uni-bielefeld.de.

** Fakultät für Mathematik, Universität Bielefeld, D-33501 Bielefeld, Germany; Department of Mathematics, Purdue University, 150 N. University St., West Lafayette, IN 47907-2067, USA.

*** Московский государственный университет им. М. В. Ломоносова, механико-математический факультет, Ленинские горы, 119992 Москва, Россия.

1) Работа выполнена при поддержке проектами РФФИ 04-01-00748, 05-01-02941-JF и 06-01-39003, HШ 1758.2003.1, DFG 436 RUS 113/343/0(R), INTAS 03-51-5018, SFB 701 при университете Билефельда и ARC_Discovery_Grant DP0663153. 
или

$$
\mathscr{L} \varphi(x, t):=\partial_{t} \varphi(x, t)+\partial_{x_{i}}\left(a^{i j}(x, t) \partial_{x_{j}} \varphi(x, t)\right)+b^{i}(x, t) \partial_{x_{i}} \varphi(x, t),
$$

где ведется суммирование по повторяющимся индексам; а интерпретация нашего уравнения состоит в следующем. Положим $A=\left(a^{i j}\right)_{i, j \leqslant d}$, $b=\left(b^{i}\right)_{i \leqslant d}$.

Будем говорить, что борелевская мера $\mu$ на $\mathbf{R}^{d}$ удовлетворяет слабому эллиптическому уравнению (1.1), если функции $a^{i j}$ и $b^{i}$ интегрируемы на каждом компактном множестве в $\mathbf{R}^{d}$ относительно меры $\mu$ и для каждого $\varphi \in C_{0}^{\infty}\left(\mathbf{R}^{d}\right)$ имеем равенство

$$
\int_{\mathbf{R}^{d}} \mathscr{L} \varphi d \mu=0
$$

понимаемое одним из следующих двух способов.

(I) Имеем $a^{i j} \in W_{\text {loc }}^{1,1}\left(\mathbf{R}^{d}\right)$, функции $a^{i j}, \partial_{x_{i}} a^{i j}$ и $b^{i}$ борелевски измеримы и локально интегрируемы относительно $|\mu|$, причем выполнено равенство

$$
\int_{\mathbf{R}^{d}}\left[a^{i j} \partial_{x_{i}} \partial_{x_{j}} \varphi+\partial_{x_{i}} a^{i j} \partial_{x_{j}} \varphi+b^{j} \partial_{x_{j}} \varphi\right] d \mu=0 .
$$

(II) Мера $\mu$ обладает плотностью $\varrho$ из класса $W_{\operatorname{loc}}^{1,1}\left(\mathbf{R}^{d}\right)$ такой, что функции $a^{i j} \partial_{x_{i}} \varrho$ и $b^{i} \varrho$ локально интегрируемы по Лебегу, причем выполнено равенство

$$
\int_{\mathbf{R}^{d}}\left[-a^{i j} \partial_{x_{i}} \varrho \partial_{x_{j}} \varphi+b^{i} \partial_{x_{i}} \varphi \varrho\right] d x=0 .
$$

Разумеется, если коэффициенты $a^{i j}$ являются локально соболевскими, а функции $\partial_{x_{i}}\left(a^{i j} \varrho\right)$ локально интегрируемы, то (1.3) можно записать как (1.2).

Аналогичным образом, борелевская мера $\mu$ на $\mathbf{R}^{d} \times(0,1)$ удовлетворяет слабому параболическому уравнению (1.1), если функции $a^{i j}$ и $b^{i}$ интегрируемы на каждом компактном множестве в $\mathbf{R}^{d} \times(0,1)$ относительно $\mu$, причем для каждой функции $\varphi \in C_{0}^{\infty}\left(\mathbf{R}^{d} \times(0,1)\right)$ имеем равенство

$$
\int_{\mathbf{R}^{d} \times(0,1)} \mathscr{L} \varphi d \mu=0,
$$

понимаемое одним из следующих двух способов.

(I) Для всякого отрезка $J \subset(0,1)$ и всякого шара $U \subset \mathbf{R}^{d}$ функции $a^{i j}$ принадлежат классу $\mathbf{H}^{1,1}(U \times J)$, функции $a^{i j}, \partial_{x_{i}} a^{i j}$ и $b^{i}$ борелевски измеримы и локально интегрируемы относительно $|\mu|$, причем выполнено равенство

$$
\int_{\mathbf{R}^{d} \times(0,1)}\left[\partial_{t} \varphi+a^{i j} \partial_{x_{i}} \partial_{x_{j}} \varphi+\partial_{x_{i}} a^{i j} \partial_{x_{j}} \varphi+b^{j} \partial_{x_{j}} \varphi\right] d \mu=0 .
$$

(II) Для всякого отрезка $J \subset(0,1)$ и всякого шара $U \subset \mathbf{R}^{d}$ ограничение меры $\mu$ на $U \times J$ имеет плотность $\varrho$ из класса $\mathbf{H}^{1,1}(U \times J)$ такую, 
что функции $a^{i j} \partial_{x_{i}} \varrho$ и $b^{i} \varrho$ локально интегрируемы по Лебегу, причем выполнено равенство

$$
\int_{\mathbf{R}^{d} \times(0,1)}\left[\partial_{t} \varphi \varrho-a^{i j} \partial_{x_{i}} \varrho \partial_{x_{j}} \varphi+b^{i} \partial_{x_{i}} \varphi \varrho\right] d x d t=0 .
$$

Ниже мы всегда указываем, какой именно случай рассматривается, посредством ссылок на (1.2) и (1.4) в случае (I) и на (1.3) и (1.5) в случае (II). В этой работе мы предполагаем, что матрицы $A(x)=$ $\left(a^{i j}(x)\right)_{i, j \leqslant d}$ и $A(x, t)=\left(a^{i j}(x, t)\right)_{i, j \leqslant d}$ симметричны и строго положительны, что гарантирует абсолютную непрерывность меры $\mu$ в случае (I). Поэтому всюду ниже мы рассматриваем меры $\mu$, представленные в виде $\mu(d t d x)=\mu_{t}(d x) d t$ посредством семейства борелевских мер $\left(\mu_{t}\right)_{t \in(0,1)}$ на $\mathbf{R}^{d}$. В этом случае (1.1) можно записать как равенство

$$
\int_{0}^{1} \int_{\mathbf{R}^{d}} \mathscr{L} \varphi(x, t) \mu_{t}(d x) d t=0
$$

понимаемое одним из двух описанных выше способов. Следует отметить, что альтернативное предположение в случае (II), что $\mu$ имеет локально соболевскую плотность, автоматически выполнено, если в случае (I) в эллиптической ситуации мы имеем $a^{i j} \in W_{\text {loc }}^{p, 1}\left(\mathbf{R}^{d}\right)$ и $b^{i} \in L_{\mathrm{loc}}^{p}\left(\mathbf{R}^{d}\right)$ с некоторым $p>d$ и функция $\operatorname{det} A$ локально отделена от нуля. Имеется похожее достаточное условие в параболическом случае. По этой причине в п. 2 мы рассматриваем решения, обладающие локально соболевскими плотностями, и не делаем предположений относительно регулярности $A$; однако в п. 3 , где речь идет о приложениях к переходным вероятностям, мы накладываем подходящие условия локальной соболевской регулярности $A$, чтобы гарантировать, что все решения удовлетворяют предположениям из п. 2.

Будем говорить, что мера $\mu=\mu_{t} d t$ удовлетворяет начальному условию $\mu_{0}=\nu$ при $t=0$, если $\nu-$ борелевская мера на $\mathbf{R}^{d}$ и

$$
\lim _{t \rightarrow 0} \int_{\mathbf{R}^{\boldsymbol{d}}} \zeta(x) \mu_{t}(d x)=\int_{\mathbf{R}^{\boldsymbol{d}}} \zeta(x) \nu(d x)
$$

для всех $\zeta \in C_{0}^{\infty}\left(\mathbf{R}^{d}\right)$.

$\mathrm{B}$ последнее десятилетие описанные уравнения привлекли значительное внимание; в работах [1]-[12] можно найти различные комментарии и обзор основных результатов, некоторые из которых будут упомянуты ниже. Основным отличием полученных в этой работе оценок от известных ранее является то, что не предполагается ограниченности или какой-либо коэрцитивности или диссипативности коэффициента сноса $b$. Например, в эллиптическом случае получена оценка

$$
\varrho(x) \geqslant C_{1} \exp \left(-C_{2}|x|^{\beta}\right)
$$

в предположении, что коэффициент диффузии $A$ равномерно ограничен вместе с $A^{-1}$, а функция $|b(x)|$ имеет мажоранту $C\left(1+|x|^{\beta}\right)$. Эта оценка 
заметно усиливает недавний важный результат работы [12], в которой накладываются значительно более сильные ограничения на коэффициенты (в частности, требуется двукратная дифференцируемость $b$ и трехкратная дифференцируемость $A$ ). Для параболического уравнения получена аналогичная, но несколько более слабая оценка

$$
\varrho(x, t) \geqslant \exp \left\{-K_{t}\left(1+|x|^{2 \beta}+|x|^{2}\right)\right\},
$$

где функция $t \mapsto K_{t}$ локально ограничена на $(0,1)$. Вместе с оценками сверху такого же вида, полученными в [10] и [11], это дает достаточно точное описание характера убывания решений на бесконечности. Доказательства основаны на предварительном исследовании зависимости постоянных в неравенстве Харнака от коэффициентов уравнения. В качестве применения даны простые достаточные условия для включения $|\nabla \varrho / \varrho|$ в $L^{p}(\mu)$ в эллиптическом случае, что усиливает важный результат работы [12]. Наконец, еще один результат нашей работы дает широкие достаточные условия существования конечной энтропии решения параболического уравнения для вероятностных мер во всякий момент времени $t_{0}>0$ с произвольным начальным распределением. Этот результат важен для исіпользвания полученных в нашей работе [10] оценок решений, поскольку в них существенно существование энтропии решения в тот момент, начиная с которого оценивается решение.

Отметим, что указанные уравнения в дивергентной форме возникают как уравнения для стационарных распределений и переходных вероятностей диффузионных процессов, задаваемых стохастическими дифференциальными уравнениями в форме Стратоновича. Для стохастического дифференциального уравнения в форме Ито

$$
d \xi_{t}=A_{0}\left(\xi_{t}\right) d w_{t}+\frac{1}{2} b\left(\xi_{t}\right) d t
$$

аналогичные уравнения записываются с недивергентным оператором

$$
L u:=a^{i j} \partial_{x_{i}} \partial_{x_{j}} u+b^{i} \partial_{x_{i}} u,
$$

где $A=A_{0} A_{0}^{T}$. Мы рассматриваем операторы дивергентного вида, что упрошает некоторые формулировки, но приводим соответствующие аналоги для недивергентных операторов.

Для произвольной области $\Omega \subset \mathbf{R}^{d}$ через $W^{q, 1}(\Omega)$ обозначим соболевское пространство функций, принадлежащих $L^{q}(\Omega)$ вместе с их обобщенными частными производными первого порядка. Это пространство наделяется стандартной нормой

$$
\|f\|_{W^{q, 1}(\Omega)}:=\|f\|_{q, 1}=\|f\|_{L^{q}(\Omega)}+\|\nabla f\|_{L^{q}(\Omega)},
$$

где $\|\cdot\|_{L^{q}(\Omega)}:=\|\cdot\|_{q}$ обозначает $L^{q}(\Omega)$-норму скалярных или векторных функций. Через $C^{0, \delta}(\Omega)$ обозначим пространство всех функций (возможно, векторных или матричных) на $\Omega$, являющихся гёльдеровыми 
порядка $\delta$. Для матричнозначного отображения $A$ на $\Omega$ положим

$$
\|A\|_{C^{0, \delta}}:=\sup _{x \in \Omega}\|A(x)\|+\sup _{x, y \in \Omega} \frac{\|A(x)-A(y)\|}{|x-y|^{\delta}} .
$$

Пусть $J \subset(0,1)$ - интервал, тогда $\|\cdot\|_{q, \Omega \times J}$ обозначает $L^{q}(\Omega \times J)$ норму скалярных или векторных функций. Наконец, $\mathbf{H}^{q, 1}(\Omega \times J)$ обозначает пространство всех измеримых функций $f$ на $\Omega \times J$ с конечной нормой

$$
\|f\|_{\mathbf{H}^{q, 1}(\Omega \times J)}:=\left(\int_{J}\left(\|f(\cdot, t)\|_{W^{q, 1}(\Omega)}\right)^{q} d t\right)^{1 / q} .
$$

Для упрощения обозначений градиент функции $u$ на $\mathbf{R}^{d} \times(0,1)$ относительно аргумента из $\mathbf{R}^{d}$ обозначается $\nabla u:=\nabla_{x} u=\left(\partial_{x_{1}} u, \ldots, \partial_{x_{d}} u\right)$. Через $U(x, R) \subset \mathbf{R}^{d}$ обозначаем открытый шар радиуса $R$ с центром в точке $x$. Пусть $|\Omega|$ - объем $\Omega$.

2. Исследование постоянных в неравенстве Харнака. В этом пункте мы исследуем зависимость постоянной в неравенстве Харнака для эллиптических и параболических уравнений от коэффициентов уравнения. Начнем с эллиптического случая. Сначала мы напомним известные априорные оценки решений эллиптических уравнений, которые будут использоваться для доказательства $p$-интегрируемости логарифмического градиента меры $\mu$.

Пусть $\Omega$ - ограниченная область в $\mathbf{R}^{d}$, функции $h^{1}, \ldots, h^{d}$ локально интегрируемы в $\Omega, x \mapsto A(x)=\left(a^{i, j}(x)\right)_{1 \leqslant i, j \leqslant d}$ - измеримое локально ограниченное на $\Omega$ матричнозначное отображение, причем матрицы $A(x)=\left(a^{i j}(x)\right)$ симметричны и положительны. Будем говорить, что функция $u$ из класса $W^{q, 1}(\Omega)$, где $q>1$, является решением уравнения

$$
\partial_{x_{i}}\left(a^{i j} \partial_{x_{j}} u+h^{i}\right)=0
$$

в $\Omega$, если для любой функции $\varphi \in C_{0}^{1}(\Omega)$ выполнено равенство

$$
\int_{\Omega}\left[\left(a^{i j} \partial_{x_{j}} u+h^{i}\right) \partial_{x_{i}} \varphi\right] d x=0
$$

Следуюшая теорема является уточнением частного случая более общего результата, который был сформулирован Ч.Б. Морри в его книге $\left[13\right.$, с. 156] не вполне корректным образом: с $\Omega^{\prime}=\Omega$, что неверно, например, для уравнения Лапласа на круге. Доказательство оценки Морри с исследованием зависимости константы от коэффициентов было дано в [14], где допущена такая же неточность, как и в книге Морри. Фактически рассуждения из [14] дают приведенную ниже оценку, а оценка с $\Omega=\Omega^{\prime}$ возможна лишь для решений с нулевым граничным условием на области с достаточно регулярной границей. Отметим, что в имеющихся применениях теоремы Морри используется 
именно доказываемое ниже верное утверждение, хотя в ряде работ оно формулируется с указанной неточностью (см., например, [3] и [6]). По этой причине мы приведем доказательство второго утверждения следующей теоремы, которое не используется в этой работе (в п. 3 будет использовано лишь хорошо известное первое утверждение с $b=0$ ).

Теорема 2.1. Предположим, что $\Omega \subset \mathbf{R}^{d}-$ ограниченная область, $A \in C^{0, \delta}(\Omega)$, где $\delta>0$, причем существует такое иисло $\alpha>0$, что $A(x) \geqslant \alpha$ Id $\partial \Omega я$ всех $x \in \Omega$. Пусть $h^{i} \in L^{q}(\Omega)$, где $q>d$. Если функиия и из $W^{q, 1}(\Omega)$ удовлетворяет уравнению (2.1) на $\Omega$, то для всякой области $\Omega^{\prime}$ с замыканием в $\Omega$ выполняется оценка

$$
\|u\|_{W^{q, 1}\left(\Omega^{\prime}\right)} \leqslant C\left(\|u\|_{L^{q}(\Omega)}+\|h\|_{L^{q}(\Omega)}\right), \quad h:=\left(h^{1}, \ldots, h^{d}\right)
$$

где число $C$ зависит только от $d, q, \alpha, \Omega, \Omega^{\prime} u\|A\|_{C^{0, \delta}}$.

Более того, если $u \in W^{q, 1}(\Omega)$ и $\partial_{x_{i}}\left(a^{i j} \partial_{x_{j}} u-b^{i} u+h^{i}\right)=0$ в том же смьсле, что и выше, причем $b^{i} \in L^{q}(\Omega)$, то выполнена более сильная оценка

$$
\|u\|_{W^{q, 1}\left(\Omega^{\prime}\right)} \leqslant C\left(\|u\|_{L^{1}(\Omega)}+\|h\|_{L^{q}(\Omega)}\right),
$$

где $C$ зависит только от $d, q, \alpha, \Omega, \Omega^{\prime},\|A\|_{C^{0, \delta}} u\left\|b^{i}\right\|_{L^{q}(\Omega)}$.

Д ок аз а тель с т о. Первое утверждение хорошо известно (см., например, [13, гл. 5]). Мы займемся более сильным вторым. Можно считать, что $A$ удовлетворяет нашим условиям на всем $\mathbf{R}^{d}$. Известно (см. [15], [16] или [17]; в последних двух работах условие на $A$ заметно ослаблено), что при $q>1$ и достаточно большом $\lambda=$ $\lambda\left(d, q, \alpha,\|A\|_{C^{0, \delta}}\right)>0$ найдется такое число $N=N\left(d, q, \alpha,\|A\|_{C^{0, \delta}}\right)>1$, что для всякой функции $w \in W^{q, 1}\left(\mathbf{R}^{d}\right)$ с компактным носителем функция $\mathscr{A} w:=\partial_{x_{i}}\left(a^{i j} \partial_{x_{j}} w\right)-\lambda w$ удовлетворяет неравенству

$$
\|w\|_{W^{q, 1}\left(\mathbf{R}^{d}\right)} \leqslant N\|\mathscr{A} w\|_{W^{q,-1}\left(\mathbf{R}^{d}\right)} .
$$

При $q>d$ по теореме вложения Соболева имеется такое число $C(d, q)>1$, что

$$
\|w\|_{L^{\infty}\left(\mathbf{R}^{d}\right)} \leqslant C(d, q) r^{1-d / q}\|\nabla w\|_{L^{q}\left(\mathbf{R}^{d}\right)} \leqslant C(d, q) r^{1-d / q}\|w\|_{W^{q, 1}\left(\mathbf{R}^{d}\right)}
$$

для всякой функции $w \in W^{q, 1}\left(\mathbf{R}^{d}\right)$ с носителем в шаре радиуса $r \leqslant 1$. Выберем шар $U=U(a, r)$ объема менее 1 с замыканием в $\Omega$ и таким радиусом $r$, что

$$
C(d, q)^{q} r^{q-d} \int_{\Omega}|b(x)|^{q} d x \leqslant(8 N)^{-q}, \quad \lambda C(d, q) r^{1-d / q} \leqslant(8 N)^{-1} .
$$

Пусть $\zeta \in C_{0}^{\infty}(U), 0 \leqslant \zeta \leqslant 1, \zeta(x)>0$ внутри $U, \zeta(x)=1$ на шаре $U(a, r / 2)$, причем

$$
J(s):=\sup _{x}|\nabla \zeta(x)| \zeta(x)^{-s}+\sup _{i, j, x}\left|\partial_{x_{i}} \partial_{x_{j}} \zeta(x)\right| \zeta(x)^{-s}<\infty, \quad 0<s<1 .
$$


Эти условия легко обеспечить, взяв $\zeta(x)=\psi(|x| / r), \psi \in C_{0}^{\infty}(\mathbf{R})$, $0 \leqslant \psi \leqslant 1, \psi(y)=0$ при $|y| \geqslant 1, \psi(y)>0$ при $|y|<1, \psi(y)=1$ при $|y| \leqslant \frac{1}{2}$ и $\psi(y)=\exp \left[\left(y^{2}-1\right)^{-1}\right]$ вблизи точек -1 и 1 . Пусть $M_{0}:=\sup _{x}\|A(x)\|$. Функция $w=\zeta u$ удовлетворяет уравнению

$$
\mathscr{A} w=-\zeta \partial_{x_{i}} h^{i}-\lambda w+\partial_{x_{i}}\left(a^{i j} u \partial_{x_{j}} \zeta+\zeta b^{i} u\right)-b^{i} u \partial_{x_{i}} \zeta+a^{i j} \partial_{x_{i}} \zeta \partial_{x_{j}} u .
$$

Нам достаточно оценить норму правой части в $W^{q,-1}(U)$ через указанные в теореме параметры, ибо затем с помощью конечного гладкого разбиения единицы получится нужная оценка. Норма первого слагаемого оценивается через $L^{q}(U)$-норму $h$ с коэффициентом, зависящим от $\zeta$. В силу нашего выбора $U$ имеем

$$
\begin{aligned}
\|\lambda w\|_{W^{q,-1}} & \leqslant \lambda\|w\|_{q} \leqslant \lambda C(d, q) r^{1-d / q}\|w\|_{q, 1} \leqslant \frac{1}{8 N}\|w\|_{q, 1}, \\
\left\|a^{i j} u \partial_{x_{j}} \zeta+\zeta b^{i} u\right\|_{q} & \leqslant M_{0}\left\|u \partial_{x_{j}} \zeta\right\|_{q}+\|\zeta u\|_{\infty}\|b\|_{q} \\
& \leqslant M_{0}\left\|u \partial_{x_{j}} \zeta\right\|_{q}+C(d, q) r^{1-d / q}\|w\|_{q, 1}\|b\|_{q} \\
& \leqslant M_{0}\left\|u \partial_{x_{j}} \zeta\right\|_{q}+\frac{1}{8 N}\|w\|_{q, 1} .
\end{aligned}
$$

Кроме того,

$$
\begin{aligned}
\left\|u \partial_{x_{j}} \zeta\right\|_{q} & =\left\|\zeta^{1-1 / q} u \zeta^{-1+1 / q} \partial_{x_{j}} \zeta\right\|_{q} \leqslant J\left(1-q^{-1}\right)\left(\int_{U}|u|^{q} \zeta^{q-1} d x\right)^{1 / q} \\
& \leqslant J\left(1-q^{-1}\right)\|\zeta u\|_{\infty}^{1-1 / q}\|u\|_{L^{1}(U)}^{1 / q} \\
& \leqslant J\left(1-q^{-1}\right) C(d, q)^{1-1 / q}\|\zeta u\|_{q, 1}^{1-1 / q}\|u\|_{L^{1}(U)}^{1 / q} .
\end{aligned}
$$

Правую часть оценим по неравенству Юнга $x^{1-1 / q} y^{1 / q} \leqslant \varepsilon x+\varepsilon^{1-q} y$ с достаточно малым $\varepsilon$ и получим оценку $\left\|a^{i j} u \partial_{x_{j}} \zeta+\zeta b^{i} u\right\|_{q} \leqslant(4 N)^{-1}\|\zeta u\|_{q, 1}+$ $C\|u\|_{L^{1}(U)}$, где $C$ выражается через $N, M_{0}, C(d, q), J(1-1 / q)$. По теореме вложения Соболева существует такое $C_{1}=C_{1}(d, q)$, что

$$
\left\|b^{i} u \partial_{x_{i}} \zeta\right\|_{W^{q,-1}(U)} \leqslant C_{1}(d, q)\left\|b^{i} u \partial_{x_{i}} \zeta\right\|_{q d /(d+q)} \leqslant C_{1}(d, q)\left\|b^{i}\right\|_{d}\left\|u \partial_{x_{i}} \zeta\right\|_{q} .
$$

Как и выше, правая часть оценивается через $(8 N)^{-1}\|w\|_{q, 1}+C_{2}\|u\|_{L^{1}(U)}$, где $C_{2}$ зависит от $d, q, M_{0}, N, \zeta,\left\|b^{i}\right\|_{L^{d}(U)}$. Итак, приходим к оценке $\|\mathscr{A} w\|_{W^{q,-1}(U)} \leqslant \frac{1}{2 N}\|w\|_{W^{q, 1}(U)}+C\left[\|\nabla u\|_{L^{d q /(d+q)}(U)}+\|u\|_{L^{1}(U)}+\|h\|_{L^{q}(U)}\right]$, что в итоге даст нужную оценку, если мы сможем оценить $L^{s}$-норму $\nabla u$, где $s=d q /(d+q)$, на шаре через $L^{1}$-норму $u$ и $L^{q}$-норму $h$ на большем шаре. Отличие от исходной задачи состоит в том, что теперь $s<d$. $\mathrm{C}$ помощью разбиения единицы достаточно оценивать $\|\nabla(\zeta u)\|_{s}$ для тех же $\zeta$, что и выше. Для $s=2$ оценка получается стандартным способом: умножим уравнение на $\zeta^{2} u$ и проинтегрируем. Это даст равенство

$$
\begin{aligned}
\int_{U}\left[a^{i j} \zeta^{2} \partial_{x_{i}} u \partial_{x_{j}} u+2 a^{i j} u \zeta \partial_{x_{i}} \zeta \partial_{x_{j}} u-b^{i} \zeta^{2} u \partial_{x_{i}} u\right. \\
\left.\quad-2 b^{i} u^{2} \zeta \partial_{x_{i}} \zeta+h^{i} \zeta^{2} \partial_{x_{i}} u+2 h^{i} u \zeta \partial_{x_{i}} \zeta\right] d x=0 .
\end{aligned}
$$


Обозначим через $I$ интеграл от $\zeta^{2}|\nabla u|^{2}$. Из оценки $A \geqslant \alpha \operatorname{Id}$ и неравенства Коши-Буняковского находим

$$
\alpha I \leqslant I^{1 / 2}\left[2 M_{0}\|u \nabla \zeta\|_{2}+\|\zeta u b\|_{2}+\|\zeta h\|_{2}\right]+\|\zeta u b\|_{2}^{2}+2\|u \nabla \zeta\|_{2}^{2}+\|\zeta h\|_{2}^{2} .
$$

Пусть $d>2$ (случай $d \leqslant 2$ проще). Пусть $l=4(d+2)^{-1}$. Ввиду вложения $W^{2,1}(U) \subset L^{2 d /(d-2)}(U)$ имеем

$$
\begin{aligned}
\|u \nabla \zeta\|_{2} & \leqslant J\left(1-\frac{l}{2}\right)\left(\int_{U}|\zeta u|^{2-l}|u|^{l} d x\right)^{1 / 2} \leqslant J\left(1-\frac{l}{2}\right)\|u\|_{L^{1}(U)}^{l / 2}\|\zeta u\|_{2 d /(d-2)}^{(2-l) / 2} \\
& \leqslant C(d, l, \varepsilon)\|u\|_{L^{1}(U)}+\varepsilon\|\zeta u\|_{2,1},
\end{aligned}
$$

$\|\zeta u b\|_{2} \leqslant\|b\|_{L^{d}(U)}\|\zeta u\|_{2 d /(d-2)} \leqslant C(d)\|b\|_{L^{d}(U)}\|\zeta u\|_{2,1}$

$$
\leqslant C(d)|U|^{(q-d) / q}\|b\|_{L^{q}(U)}\|\zeta u\|_{2,1} .
$$

Уменьшив $r$, получим $C(d)|U|^{(q-d) / q}\|b\|_{L^{q}(U)} \leqslant \alpha^{1 / 2} / 2$. Выбрав $\varepsilon$ достаточно малым, приходим к оценке $\|\zeta u\|_{2,1} \leqslant C\left[\|h\|_{2}+\|u\|_{L^{1}(U)}\right]$, где $C$ зависит от $d, q, \zeta, \alpha, M_{0},\|b\|_{q}$. Несколько итераций с помощью теоремы вложения позволяют перейти от $s=2$ к $s=d q /(d+q)$. В самом деле, если при некотором $s \in[2, d)$ есть оценка $\|\zeta u\|_{W^{s, 1}(U)} \leqslant C\left[\|u\|_{L^{1}(U)}+\|h\|_{L^{q}(U)}\right]$, то через правую часть оценивается $\|\zeta u\|_{s d /(d-s)}$. В меньшем шаре это дает $\|w\|_{t, 1} \leqslant N\|\mathscr{A} w\|_{W^{t,-1}} \leqslant C^{\prime}\left[\|u\|_{L^{1}(U)}+\|h\|_{L^{q}(U)}\right]$, где

$$
t=\frac{q d s}{q d-q s+d s} \geqslant \theta s \quad \text { и } \quad \theta=\left(1-\frac{s}{d}+\frac{s}{q}\right)^{-1} \geqslant\left(1-\frac{1}{d}+\frac{1}{q}\right)^{-1}>1,
$$

поскольку $\|u b\|_{t} \leqslant\|b\|_{q}\|u\|_{s d /(d-s)},\|u\|_{t} \leqslant\|u\|_{s d /(d-s)}$ и $\|\nabla u\|_{W^{t,-1}} \leqslant$ $C(t, d)\|\nabla u\|_{t d /(t+d)} \leqslant C(t, d)\|\nabla u\|_{s}$ ввиду соотношения $t d /(t+d)=q s /(q+$ $s)<s$. Теорема доказана.

Следствие 2.1. Если $\Omega=U(z, R)$, где $R<1$, то при $\lambda<1$ в первом утверждении теоремь 2.1 выполняется оченка

$$
\|u\|_{W^{q, 1}(U(z, \lambda R))} \leqslant R^{-1} C\left(\|u\|_{L^{q}(U(z, R))}+R\|h\|_{L^{q}(U(z, R))}\right)
$$

где $C=C\left(d, q, \alpha,\|A\|_{\left.C^{0, \delta}, \lambda\right)}\right.$.

Д ок аз а т е л ь с т в о. Сделаем замену координат $x=z+R y$. Функция $v(y)=u(z+R y)$ удовлетворяет в $U(0,1)$ уравнению

$$
\partial_{y_{j}}\left(a^{i j}(z+R y) \partial_{y_{i}} v(y)+R h^{i}(z+R y)\right)=0 .
$$

По условию матрица $A$ гёльдерова, и можно считать, что постоянная Гёльдера не изменилась, так как $R<1$. Тогда для функции $v$ имеет место оценка

$$
\|v\|_{W^{q, 1}(U(0, \lambda))} \leqslant C\left(d, q, \alpha,\|A\|_{\left.C^{0, \delta}, \lambda\right)\left(\|v\|_{L^{q}(U(0,1))}+R\|h\|_{L^{q}(U(0,1))}\right) .}\right.
$$

Сделав обратную замену координат и учтя, что $R<1$, получим

$$
\begin{aligned}
\|v\|_{W^{q, 1}(U(0, \lambda))} & =R^{-d / q}\|u\|_{L^{q}(U(z, \lambda R))}+R^{1-d / q}\|\nabla u\|_{L^{q}(U(z, \lambda R))} \\
& \geqslant R^{1-d / q}\|u\|_{W^{q, 1}(U(z, \lambda R))} .
\end{aligned}
$$


Аналогично имеем

$$
\begin{aligned}
\|v\|_{L^{q}(U(0,1))}+R\|h\|_{L^{q}(U(0,1))} & =R^{-d / q}\|u\|_{L^{q}(U(z, R))}+R^{1-d / q}\|h\|_{L^{q}(U(z, R))} \\
& =R^{-d / q}\left(\|u\|_{L^{q}(U(z, R))}+R\|h\|_{L^{q}(U(z, R))}\right) .
\end{aligned}
$$

На основании этих оценок получаем

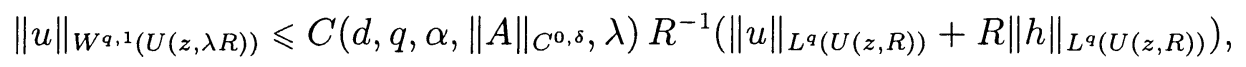

что и требовалось. Следствие доказано.

Далее будем предполагать, что существуют такие постоянные $\gamma \geqslant 0$ и $\alpha>0$, что

$$
\sum_{i, j}\left|a^{i j}(x)\right|^{2} \leqslant \gamma^{2} \quad \text { и } \quad A(x) \geqslant \alpha \text { Id } \quad \text { для всех } x \in \Omega .
$$

Пусть отображение $b: \Omega \rightarrow \mathbf{R}^{d}$ измеримо и

$$
\sup _{x \in \Omega}|b(x)| \leqslant B<\infty .
$$

Предположим, что неотрицательная функция $u \in W^{2,1}(\Omega)$ удовлетворяет уравнению

$$
\partial_{x_{i}}\left(a^{i j} \partial_{x_{j}} u-b^{i} u\right)=0,
$$

т.е. для всякой функции $\varphi \in C_{0}^{1}(\Omega)$ выполнено равенство

$$
\int_{\Omega} \partial_{x_{i}} \varphi\left(a^{i j} \partial_{x_{j}} u-b^{i} u\right) d x=0 .
$$

При указанных условиях функция $u$ имеет локально гёльдерову версию, в частности, эта версия локально ограничена на $\Omega$ (см. [19, теорема 8.22]).

Для всякой интегрируемой на $\Omega$ функции $v$ положим

$$
v_{\Omega}=\frac{1}{|\Omega|} \int_{\Omega} v d x
$$

В нашем исследовании постоянных в неравенстве Харнака для решений уравнения (2.3) придется воспроизвести основные этапы доказательства теоремы 8.18 в [19]. Отметим, что в замечании к теореме 8.20 из [19] указана нужная нам зависимость константы от коэффициентов уравнения, но без обоснования. Поскольку для дальнейшего важен точный вид этой зависимости, мы даем полное обоснование.

Справедлива следующая лемма (см. [19, лемма 7.21]).

Лемма 2.1. Пусть $\Omega$ - выпуклая область, и пусть $v \in W^{1,1}(\Omega)$. Предположим, что существует такая постоянная $K$, что для каждого шара $U\left(x_{0}, R\right)$ выполнено следуюшее неравенство:

$$
\int_{\Omega \cap U\left(x_{0}, R\right)}|\nabla v| d x \leqslant K R^{d-1} .
$$


Тогда найдутся такие положительные постоянные $\sigma_{0} u$, зависящие лишь om $d$, что

$$
\int_{\Omega} \exp \left(\frac{\sigma}{K}\left|v-v_{\Omega}\right|\right) d x \leqslant C(\operatorname{diam} \Omega)^{d}
$$

где $\sigma=\sigma_{0}|\Omega|(\operatorname{diam} \Omega)^{-d} u \operatorname{diam} \Omega=\sup _{x, y \in \Omega}|x-y|$.

Теорема 2.2. Пусть $\theta=1+4 \delta$, где $0<\delta \leqslant 3$. Eсли $U(y, \theta R) \subset \Omega$, то выполнено следующее неравенство для непрерывной версии функиии $и$ :

$$
\sup _{x \in U(y, R)} u(x) \leqslant C \inf _{x \in U(y, R)} u(x)
$$

$2 \partial e$

$$
C=\exp \left\{c(d) \delta^{-1}\left(\gamma \alpha^{-1}+B \alpha^{-1} R\right)\right\},
$$

причем число $c(d)$ зависит лишь от $d$.

Д о к а з а т е л ь с т в о. Можно считать, что $d \geqslant 3$. Случай $d \leqslant 2$ сводится к случаю $d=3$, если считать $u$ функцией на $\mathbf{R}^{3}$ и положить $b^{3}=0, a^{33}=1, a^{32}=a^{23}=a^{31}=a^{13}=0$.

1. Пусть $R=1, y=0$. Пусть $\eta \in C_{0}^{1}(\Omega)$ и $u_{\varepsilon}=u+\varepsilon, \varepsilon>0$. Пусть $\beta \in(-\infty,+\infty)$. Легко видеть, что (2.4) остается в силе и для $\varphi=\eta^{2} u_{\varepsilon}^{\beta}$, откуда получаем

$$
\begin{aligned}
\beta \int_{\Omega}\left(A \nabla u, \nabla u_{\varepsilon}\right) u_{\varepsilon}^{\beta-1} \eta^{2} d x= & -2 \int_{\Omega}(A \nabla u, \nabla \eta) u_{\varepsilon}^{\beta} \eta d x \\
& +\beta \int_{\Omega}\left(u b, \nabla u_{\varepsilon}\right) u_{\varepsilon}^{\beta-1} \eta^{2} d x+2 \int_{\Omega}(u b, \nabla \eta) u_{\varepsilon}^{\beta} \eta d x .
\end{aligned}
$$

Применяя неравенство Коши и принимая во внимание наши предположения относительно коэффициентов, получаем

$$
\int_{\Omega}\left|\nabla u_{\varepsilon}\right| u_{\varepsilon}^{\beta-1} \eta^{2} d x \leqslant C \int_{\Omega}(\eta+|\nabla \eta|)^{2} u_{\varepsilon}^{\beta+1} d x
$$

где

$$
C=4\left(\left(\frac{\gamma}{|\beta| \alpha}+\frac{B}{\alpha}\right)^{2}+\frac{B}{|\beta| \alpha}\right)
$$

Так как $\alpha \leqslant \gamma$, то имеем

$$
C \leqslant 8\left(\frac{\gamma}{|\beta| \alpha}+\frac{B}{\alpha}\right)^{2} .
$$

Поэтому будем использовать оценку (2.6) с $C=8\left(\gamma|\beta|^{-1} \alpha^{-1}+B \alpha^{-1}\right)^{2}$.

Пусть $w=u_{\varepsilon}^{(\beta+1) / 2}$ при $\beta \neq-1$ и $w=\ln u_{\varepsilon}$ при $\beta=-1$. Если $\beta=-1$, то имеем

$$
\int_{\Omega}|\nabla w|^{2} \eta^{2} d x \leqslant C_{1} \int_{\Omega}(\eta+|\nabla \eta|)^{2} d x
$$

где

$$
C_{1}=8\left(\frac{\gamma}{\alpha}+\frac{B}{\alpha}\right)^{2}
$$


Если $\beta \neq-1$, то

$$
\int_{\Omega}|\nabla w|^{2} \eta^{2} d x \leqslant C_{2} \int_{\Omega}(\eta+|\nabla \eta|)^{2} w^{2} d x
$$

где

$$
C_{2}=2(\beta+1)^{2}\left(\frac{\gamma}{|\beta| \alpha}+\frac{B}{\alpha}\right)^{2}
$$

2. Положим

$$
F(p, r)=\left(\int_{U(0, r)} u_{\varepsilon}^{p} d x\right)^{1 / p}, \quad p \neq 0 .
$$

Мы найдем числа $p_{0}=p_{0}(d, \delta, \alpha, \gamma, B)>0$ и $C_{3}=C_{3}(d, \delta, \alpha, \gamma, B)>0$, такие, что

$$
F\left(p_{0}, 1+3 \delta\right) \leqslant C_{3} F\left(-p_{0}, 1+3 \delta\right) .
$$

Пусть $r \leqslant 1, U_{r+\delta r}-$ произвольный шар радиуса $r+\delta r$, содержащийся в $U(0, \theta)$, и пусть $U_{r}$ - шар радиуса $r$ с тем же центром, что и $U_{r+\delta r}$. Возьмем такую гладкую функцию $\eta$, что $\eta(x)=1$ при $x \in U_{r}, \eta(x)=0$ при $x \notin U_{r+\delta r}$, причем $0 \leqslant \eta \leqslant 1$ и $|\nabla \eta| \leqslant 2(\delta r)^{-1}$. Согласно оценке $(2.7)$ с $\beta=-1$, получаем

$$
\int_{U_{r}}|\nabla w| d x \leqslant(2 r)^{d / 2}\left(\int_{U_{r}}|\nabla w|^{2} d x\right)^{1 / 2} \leqslant K_{0}(d) \delta^{-1} C_{1}^{1 / 2} r^{d-1} .
$$

По лемме 2.1 существуют такие числа $C(d)$ и $K(d)$, зависящие только от $d$, что выполняется следующее неравенство:

$$
\int_{U(0,1+3 \delta)} \exp \left(p_{0}\left|w-w_{U(0,1+3 \delta)}\right|\right) d x \leqslant K(d), \quad p_{0}=C(d) \delta C_{1}^{-1 / 2} .
$$

Следовательно, имеем

$$
\begin{aligned}
& \int_{U(0,1+3 \delta)} e^{p_{0} w} d x \int_{U(0,1+3 \delta)} e^{-p_{0} w} d x \\
& \quad \leqslant \int_{U(0,1+3 \delta)} e^{p_{0} w-w_{U(0,1+3 \delta)}} d x \int_{U(0,1+3 \delta)} e^{w_{U(0,1+3 \delta)}-p_{0} w} d x \leqslant K(d)^{2} .
\end{aligned}
$$

Вспоминая, что $w=\ln u_{\varepsilon}$, получаем оценку

$$
F\left(p_{0}, 1+3 \delta\right) \leqslant C_{3} F\left(-p_{0}, 1+3 \delta\right),
$$

где

$$
C_{3}=K(d)^{2 / p_{0}}=\exp \left(c_{1}(d) \delta^{-1} \alpha^{-1}(\gamma+B)\right),
$$

а число $c_{1}(d)$ зависит только от $d$.

3. Известно, что

$$
\begin{aligned}
& F(+\infty, 1):=\sup _{U(0,1)} u_{\varepsilon}=\lim _{p \rightarrow+\infty} F(p, 1), \\
& F(-\infty, 1):=\inf _{U(0,1)} u_{\varepsilon}=\lim _{p \rightarrow-\infty} F(p, 1) .
\end{aligned}
$$


Мы найдем числа $C_{4}=C_{4}(d, \delta, \alpha, \gamma, B)>0$ и $C_{5}=C_{5}(d, \delta, \alpha, \gamma, B)>0$ такие, что

$$
F(+\infty, 1) \leqslant C_{4} F\left(p_{0}, 1+3 \delta\right) \leqslant C_{3} F\left(-p_{0}, 1+3 \delta\right) \leqslant C_{5} F(-\infty, 1) .
$$

По неравенству треугольника имеем

$$
\left(\int_{\Omega}|\nabla(\eta w)|^{2} d x\right)^{1 / 2} \leqslant\left(\int_{\Omega}|\eta \nabla w|^{2} d x\right)^{1 / 2}+\left(\int_{\Omega}|w \nabla \eta|^{2} d x\right)^{1 / 2} .
$$

Согласно оценке $(2.8)$ с $\beta \neq-1$, получаем

$$
\left(\int_{\Omega}|\nabla(\eta w)|^{2} d x\right)^{1 / 2} \leqslant 2\left(C_{2}+1\right)^{1 / 2}\left(\int_{\Omega}(\eta+|\nabla \eta|)^{2} w^{2} d x\right)^{1 / 2} .
$$

Пусть гладкая функция $\eta$ такова, что $\eta(x)=1$ при $|x| \leqslant r_{1}, \eta(x)=0$ при $|x| \geqslant r_{2}$, где $1<r_{1}<r_{2} \leqslant 1+3 \delta$, причем $0 \leqslant \eta \leqslant 1$ и $|\nabla \eta| \leqslant 2 /\left(r_{2}-r_{1}\right)$. По теореме вложения Соболева неравенство (2.9) дает оценку

$$
\left(\int_{U\left(0, r_{1}\right)} w^{2 d /(d-2)} d x\right)^{(d-2) /(2 d)} \leqslant \frac{C_{0}(d)\left(C_{2}+1\right)^{1 / 2}}{r_{2}-r_{1}}\left(\int_{U\left(0, r_{2}\right)} w^{2} d x\right)^{1 / 2},
$$

где число $C_{0}(d)$ зависит только от $d$. Пусть $\beta+1>0$. Так как $w=u_{\varepsilon}^{(\beta+1) / 2}$, то мы имеем

$$
F\left(\frac{d}{d-2}(\beta+1), r_{1}\right) \leqslant\left(\frac{C_{0}(d)\left(C_{2}+1\right)^{1 / 2}}{r_{2}-r_{1}}\right)^{2 /(\beta+1)} F\left(\beta+1, r_{2}\right) .
$$

Положим $q=d /(d-2)>1$. Мы будем применять полученную оценку к числам $r_{1}$ и $r_{2}$ вида $r_{1}=r_{m}=1+\delta 2^{-m}, r_{2}=r_{m-1}=1+\delta 2^{-m+1}$, причем будем брать в качестве $\beta+1$ числа $q^{m} p$, где $m=0,1,2, \ldots$, а число $p$ задано равенством

$$
p=d(d-1)^{-1}\left((d-2) d^{-1}\right)^{k},
$$

в котором $k$ - такое целое число, что

$$
\frac{\ln p_{0}-\ln d+\ln (d-1)}{\ln (d-2)-\ln d}<k<\frac{\ln p_{0}-\ln d+\ln (d-1)}{\ln (d-2)-\ln d}+2 .
$$

Заметим, что

$$
(d-2)^{2} d^{-2} p_{0}<p<p_{0} .
$$

Кроме того, для всех неотрицательных целых чисел $m$ имеет место оценка

$$
\left|q^{m} p-1\right|=\left|d(d-1)^{-1}\left(1-2 d^{-1}\right)^{k-m}-1\right| \geqslant(d-1)^{-1},
$$

которая очевидна из рассмотрения случаев $k \geqslant m$ и $k<m$. Из этой оценки следует, что $C_{2} \leqslant 2(d-1)^{2}(\beta+1)^{2} \alpha^{-2}(\gamma+B)^{2}$, откуда ввиду соотношения $p<p_{0}$ мы получаем неравенство

$$
C_{2} \leqslant 2(d-1)^{2} q^{2 m} p_{0}^{2}\left(\frac{\gamma}{\alpha}+\frac{B}{\alpha}\right)^{2}=2 \delta^{2} C(d)^{2}(d-1)^{2} q^{2 m} .
$$


Итак, приходим к соотношению

$$
F\left(q^{m+1} p, r_{m}\right) \leqslant\left(Q(d) \delta^{2}\right)^{2 m /\left(q^{m} p\right)} F\left(q^{m} p, r_{m-1}\right),
$$

где число $Q(d)$ зависит только от $d$. Это соотношение дает оценку

$$
F\left(q^{m} p, r_{m}\right) \leqslant\left(Q(d) \delta^{2}\right)^{S / p} F(p, 1+\delta), \quad S:=S(d):=2 \sum_{m=1}^{\infty} m q^{-m} .
$$

По неравенству Гёльдера имеем $F(p, 1+\delta) \leqslant \exp \left(c_{2}(d) / p_{0}\right) F\left(p_{0}, 1+3 \delta\right)$. Наконец, получаем неравенство $F(+\infty, 1) \leqslant C_{4} F\left(p_{0}, 1+3 \delta\right)$, где

$$
C_{4}=\exp \left\{c_{3}(d) \delta^{-1} \alpha^{-1}(\gamma+B)\right\} .
$$

Если $\beta+1<0$, таким же образом имеем

$$
F\left(\beta+1, r_{2}\right) \leqslant\left(\frac{C_{0}(d)\left(C_{2}+1\right)^{1 / 2}}{r_{2}-r_{1}}\right)^{2 /|\beta+1|} F\left(\frac{d}{d-2}(\beta+1), r_{1}\right) .
$$

Повторяя предыдущее рассуждение, получаем оценку

$$
F\left(-p_{0}, 1+3 \delta\right) \leqslant C_{5} F(-\infty, 1), \quad C_{5}=\exp \left\{c_{4}(d) \delta^{-1} \alpha^{-1}(\gamma+B)\right\} .
$$

Вместе с нашими предыдущими оценками это дает (2.5) для $u_{\varepsilon}$ вместо $u$ с постоянной требуемого вида, не зависящей от $\varepsilon$, в случае $R=1, y=0$. Полагая $\varepsilon \rightarrow 0$, получаем нужную оценку и для $u$. Случай $R \neq 1$ сводится к рассмотренному случаю заменой переменных $x=y+R z$. Функция $v(z)=u(y+z R)$ удовлетворяет уравнению $\partial_{z_{i}}\left(a^{i j} \partial_{z_{j}} v-R b^{i} v\right)=0$. Поэтому $B$ надо заменить на $B R$, а постоянные $\gamma$ и $\alpha$ оставить без изменения. Теорема доказана.

Следует отметить, что в задаче 8.3 на с. 206 в [19] ошибочно утверждается, что в случае, когда матрица $A$ симметрична (как мы и предполагаем), постоянная может быть уточнена следующим образом: $C=\exp \left(c(d)(\gamma / \alpha+(B / \alpha) R)^{1 / 2}\right)$. Однако это неверно в случае, когда $A=\mathrm{Id}$ и $b(x)=-x$, поскольку решение является гауссовским и не может оцениваться снизу через $C_{1} \exp \left(-C_{2}|x|\right)$.

3 а м е ч а н и е 2.1. В случае, когда наш эллиптический оператор записан в недивергентной форме $L=a^{i j} \partial_{x_{i}} \partial_{x_{j}}+b^{i} \partial_{x_{i}}$, причем $A$ локально липшицево, такая же нижняя оценка справедлива, если заменить $b^{i}$ на $b^{i}-\partial_{x_{j}} a^{i j}$.

Теперь рассмотрим неравенство Харнака для параболического уравнения. Пусть $\Omega$ - ограниченная область в $\mathbf{R}^{d}, Q=\Omega \times(0,1)$, $A=\left(a^{i j}\right)_{1 \leqslant i, j \leqslant d}$ - измеримое матричнозначное отображение на $Q$, $a^{i j}=a^{j i}$, причем существуют такие постоянные $\gamma \geqslant 0$ и $\alpha>0$, что

$$
\sum_{i, j}\left|a^{i j}(x, t)\right|^{2} \leqslant \gamma^{2} \quad \text { и } \quad A(x, t) \geqslant \alpha \text { Id } \quad \text { для всех }(x, t) \in Q .
$$

Кроме того, пусть $b: Q \rightarrow \mathbf{R}^{d}-$ измеримое векторное поле и

$$
\sup _{(x, t) \in Q}|b(x, t)| \leqslant B<\infty .
$$


Предположим, что неотрицательная функция $u \in \mathbf{H}^{2,1}(Q)$ является решением уравнения

$$
\partial_{t} u=\partial_{x_{i}}\left(a^{i j} \partial_{x_{j}} u-b^{i} u\right)
$$

т.е. для всякой функции $\varphi \in C_{0}^{1}(Q)$ выполнено равенство

$$
\iint_{Q}\left[-\partial_{t} \varphi u+\partial_{x_{i}} \varphi\left(a^{i j} \partial_{x_{j}} u-b^{i} u\right)\right] d x d t=0 .
$$

Из общей теории параболических уравнений (см., например, [18, гл. 3, теорема 8.1 в $\S 8$ и теорема 10.1 в $\S 10]$ ) следует, что при наших предположениях всякое решение $u$ имеет локально гёльдерову версию.

Зафиксируем точку $(\bar{x}, \bar{t}) \in \Omega \times(0,1]$. Пусть $R(\bar{x}, r)$ - открытый куб с центром в точке $\bar{x}$ и длиной ребра $r$. Положим

$$
Q(r)=R(\bar{x}, r) \times\left(\bar{t}-r^{2}, \bar{t}\right), \quad Q^{*}(r)=R(\bar{x}, r) \times\left(\bar{t}-8 r^{2}, \bar{t}-7 r^{2}\right) .
$$

Имеет место следующая классическая теорема (см. теорему 3 в [20], усиливающую результат из [21]).

Теорема 2.3. Пусть $Q(3 r) \subset Q$. Тогда для непрерьвной версии функции и, удовлетворяющей уравнению (2.11), имеем

$$
\sup _{(x, t) \in Q^{*}(r)} u(x, t) \leqslant C \inf _{(x, t) \in Q(r)} u(x, t)
$$

где число $C=C(d, \alpha, \gamma, B r)$ зависит лишь от $d ; \alpha, \gamma$ u $B r$.

Как и в эллиптическом случае, мы интересуемся более точным видом зависимости $C$ от указанных параметров.

Пусть $Q^{\prime}=\Omega^{\prime} \times J^{\prime}$, где $\Omega^{\prime} \subset \Omega$ открыто и $J^{\prime} \subset[0,1]-$ некоторый интервал. Если $p, q>1$, то положим

$$
\|f\|_{p, q, Q^{\prime}}:=\left(\int_{J^{\prime}}\left(\int_{\Omega^{\prime}}|f(x, t)|^{p} d x\right)^{q / p} d t\right)^{1 / q} .
$$

Если $q=\infty$, то положим

$$
\|f\|_{p, \infty, Q^{\prime}}:=\sup _{J^{\prime}}\|f(\cdot, t)\|_{L^{p}\left(\Omega^{\prime}\right)} .
$$

Пусть $L^{2, \infty}\left(Q^{\prime}\right)$ обозначает пространство всех таких измеримых функций $f$ на $Q^{\prime}$, что $\|f\|_{2, \infty, Q^{\prime}}<\infty$. Доказательство следующей леммы можно найти в $[18$, гл. $2, \S 3]$.

Лемма 2.2. Пусть $d>2$. Для всякой функиии $v \in \mathbf{H}^{2,1}\left(Q^{\prime}\right) \bigcap$ $L^{2, \infty}\left(Q^{\prime}\right)$ такой, что при почти всех $t \in J^{\prime}$ функиия $x \mapsto v(x, t)$ имеет компактный носитель в $\Omega^{\prime}$, имеет место неравенство

$$
\|v\|_{p, q, Q^{\prime}} \leqslant c(d, p)\left(\|\nabla v\|_{L^{2}\left(Q^{\prime}\right)}+\|v\|_{2, \infty, Q^{\prime}}\right),
$$

где $2 \leqslant q, 2 \leqslant p \leqslant 2 d /(d-2)$ u $1 / q+d /(2 p)=d / 4$. 
3 а м е ч а н и е 2.2. Выделим важный частный случай этой леммы, который будет использован ниже. Если $p=q=2(d+2) / d$, то неравенство выше приобретает вид

$$
\|u\|_{L^{2(d+2) / d}\left(Q^{\prime}\right)} \leqslant C\left(\|\nabla u\|_{L^{2}\left(Q^{\prime}\right)}+\|u\|_{2, \infty, Q^{\prime}}\right),
$$

где $C$ зависит только от $d$.

Положим $u_{\varepsilon}=u+\varepsilon$ при $\varepsilon>0$ и

$$
\mathscr{H}= \begin{cases}\frac{1}{\beta+1} u_{\varepsilon}^{\beta+1}, & \beta \neq-1, \\ \ln u_{\varepsilon}, & \beta=-1 .\end{cases}
$$

Лемма 2.3. Пусть $\eta \in C_{0}^{1}(Q)$. Для почти всех $\tau_{1}, \tau_{2} \in(0,1)$ имеем

$$
\begin{aligned}
& \operatorname{sign} \beta\left(\int_{\Omega}\left[\eta\left(x, \tau_{2}\right)^{2} \mathscr{H}\left(x, \tau_{2}\right)-\eta\left(x, \tau_{1}\right)^{2} \mathscr{H}\left(x, \tau_{1}\right)\right] d x\right. \\
& \left.\quad+\frac{\alpha \beta}{2} \int_{\tau_{1}}^{\tau_{2}} \int_{\Omega} \eta^{2} u_{\varepsilon}^{\beta-1}\left|\nabla u_{\varepsilon}\right|^{2} d x d t\right) \\
& \leqslant C(\alpha, \gamma, \beta) \int_{\tau_{1}}^{\tau_{2}} \int_{\Omega}(|\nabla \eta|+\eta)^{2} u_{\varepsilon}^{\beta+1} d x d t+2 \int_{\tau_{1}}^{\tau_{2}} \int_{\Omega} \eta\left|\partial_{t} \eta \| \mathscr{H}\right| d x d t
\end{aligned}
$$

¿de

$$
C(\alpha, \gamma, \beta)=\frac{8}{\alpha|\beta|}(|\beta| B+\gamma)^{2} .
$$

Д ок а за т ель с т в о. Достаточно повторить рассуждения из разделов 2 и 9 в [20], где надо положить $f=g=h=0$ и учесть, что $\gamma \geqslant \alpha$.

Пусть, как и выше, точка $(\bar{x}, \bar{t}) \in \Omega \times(0,1]$ фиксирована и $R(r):=$ $R(\bar{x}, r)$ - открытый куб с длиной ребра $r$ и центром в точке $\bar{x}$. Пусть $D:=R\left(\frac{1}{2}\right) \times(0,1)$, и пусть $Q^{+}(l)$ и $Q^{-}(l)-$ прямоугольники, содержащиеся в $D$ и полученные из $R\left(\frac{1}{2}\right) \times\left(0, \frac{1}{2}\right)$ и $R\left(\frac{1}{2}\right) \times\left(\frac{1}{2}, 1\right)$ соответственно посредством преобразований вида

$$
t \mapsto l^{2} t+c_{1}, \quad x \mapsto l x+c_{2}
$$

с некоторыми числами $c_{1}, c_{2}, l$. Положим $\psi(w)=\sqrt{w}$ при $w>0$ и $\psi(w)=0$ при $w \leqslant 0$.

Лемма 2.4. Пусть $D \subset Q$. Для всех $Q^{+}(l), Q^{-}(l)$ имеем

$$
\iint_{Q^{-}(l)} \iint_{Q^{+}(l)} \psi\left(\ln \frac{u_{\varepsilon}(y, s)}{u_{\varepsilon}(x, t)}\right) d y d s d x d t \leqslant B_{0} l^{2 d+4}
$$

где

$$
\begin{aligned}
B_{0} & :=C(d)\left(1+\alpha^{-1}+\alpha^{-1 / 2}(B+\gamma)\right) \\
& \leqslant B_{1}:=C(d)\left(1+\alpha^{-1}+\left(\alpha^{-1 / 2}+\alpha^{-1}\right)(B+\gamma)\right) .
\end{aligned}
$$


Д о к а зате ль с т о. Подробное доказательство дано в разделе 6 работы [20]. Для полного соответствия достаточно заметить, что указанная там постоянная $C_{6}$ в нашем случае имеет вид $C_{6}=4 C(\alpha, \gamma)=$ $32 \alpha^{-1}(B+\gamma)^{2}$.

Пусть $D^{-}=R\left(\frac{1}{2}\right) \times\left(0, \frac{1}{4}\right)$ и $D^{+}=R\left(\frac{1}{2}\right) \times\left(\frac{3}{4}, 1\right)$. Тогда лемма 2.4 и хорошо известная лемма Мозера [21] дают следующее утверждение.

Следствие 2.2. Пусть $D \subset Q$. Тогда выполнено следуюшее неравенство:

$$
\iint_{D^{-}} u_{\varepsilon}^{\lambda / B_{1}^{2}} d y d s \iint_{D^{+}} u_{\varepsilon}^{-\lambda / B_{1}^{2}} d x d t \leqslant K
$$

где $B_{1}$ - постоянная из леммь 2.4 , а числа $\lambda=\lambda(d)$ u $K=K(d)$ зависят только от $d$.

Теорема 2.4. Пусть $Q(3 r) \subset Q$. Тогда для непрерьвной версии функции и выполнено следующее неравенство:

$$
\sup _{(x, t) \in Q^{*}(r)} u(x, t) \leqslant C \inf _{(x, t) \in Q(r)} u(x, t),
$$

где

$$
C:=C(d, \alpha, \gamma, B, r):=\exp \left\{c(d)\left[1+\alpha^{-1}+\left(\alpha^{-1 / 2}+\alpha^{-1}\right)(B r+\gamma)\right]^{2}\right\} .
$$

Д ок а з а т льство. Будем следовать доказательству теоремы 3 в [20].

1. Пусть $\bar{x}=0, \bar{t}=1, r=\frac{1}{3}$ и $Q(3 r)=R(1) \times(0,1) \subset Q$. Как и выше, положим $u_{\varepsilon}=u+\varepsilon, \varepsilon>0$. Достаточно получить оценку

$$
\sup _{(x, t) \in Q^{*}\left(\frac{1}{3}\right)} u_{\varepsilon}(x, t) \leqslant C \inf _{(x, t) \in Q\left(\frac{1}{3}\right)} u_{\varepsilon}(x, t), \quad C=C\left(d, \alpha, \gamma, B, \frac{1}{3}\right) .
$$

Если $\frac{1}{3} \leqslant s \leqslant \frac{1}{2}$, то положим $S(s)=R(s) \times((1-s) / 6,(1+s) / 6)$. Пусть $l$ и $l^{\prime}-$ такие два числа, что $\frac{1}{3}<l^{\prime}<l<\frac{1}{2}$, и пусть гладкая функция $\eta$ такова, что $\eta(x, t)=1$ при $(x, t) \in S\left(l^{\prime}\right), \eta(x, t)=0$ вне $S(l)$ и

$$
0 \leqslant \eta \leqslant 1, \quad|\nabla \eta| \leqslant 2\left(l-l^{\prime}\right)^{-1}, \quad\left|\partial_{t} \eta\right| \leqslant 6\left(l-l^{\prime}\right)^{-1} .
$$

Пусть $\beta>-1$ и $v=u_{\varepsilon}^{(\beta+1) / 2}$. Согласно лемме 2.3 , имеем

$$
\|\eta \nabla v\|_{L^{2}(S(l))}^{2} \leqslant C_{1}\|v\|_{L^{2}(S(l))}^{2}, \quad\|\eta v\|_{2, \infty}^{2} \leqslant C_{2}\|v\|_{L^{2}(S(l))}^{2},
$$

где

$$
C_{1}=C(d) \frac{(\beta+1)^{2}}{\left(l-l^{\prime}\right)^{2}}\left(\frac{B}{\alpha}+\frac{\gamma}{|\beta| \alpha}\right)^{2}, \quad C_{2}=\frac{\alpha|\beta| C_{1}}{(\beta+1)} .
$$

Применяя замечание 2.2 , получаем

$$
\|v\|_{L^{2(d+2) / d}\left(S\left(l^{\prime}\right)\right)} \leqslant\left(C_{3}+1\right)^{1 / 2}\|v\|_{L^{2}(S(l))},
$$

где

$$
C_{3}=C(d) \frac{(\beta+1)(\beta+1+\alpha|\beta|)}{\left(l-l^{\prime}\right)^{2}}\left(\frac{B}{\alpha}+\frac{\gamma}{|\beta| \alpha}\right)^{2} .
$$


Как и в эллиптическом случае, если $p>0$, то положим

$$
F(p, l)=\left(\iint_{S(l)} u_{\varepsilon}^{p} d x d t\right)^{1 / p} .
$$

Мы доказали, что если $\beta+1>0$ и $q=(d+2) / d$, то выполнено следующее неравенство:

$$
F\left(q(\beta+1), l^{\prime}\right) \leqslant\left(C_{3}+1\right)^{1 /(\beta+1)} F(\beta+1, l) .
$$

Положим $l=l_{m}=3^{-1}\left(1+2^{-m-1}\right), l^{\prime}=l_{m}^{\prime}=3^{-1}\left(1+2^{-m-2}\right)$ и $p_{0}=\lambda / B_{1}^{2}$, где $\lambda$ - постоянная из следствия 2.2 , а число $B_{1}$ определено в лемме 2.4 . Существует такое число $p$, удовлетворяющее условию $(d /(d+2))^{2} p_{0}<$ $p<p_{0}$, что для некоторой постоянной $C(d)>1$, зависящей только от размерности $d$, при всех $m$ мы имеем

$$
\begin{aligned}
C_{3} & \leqslant C(d) \frac{q^{2 m} p_{0}^{2}(1+\alpha)}{\left(l-l^{\prime}\right)^{2}}\left(\frac{B}{\alpha}+\frac{\gamma}{\alpha}\right)^{2} \leqslant C(d) \frac{q^{2 m} p_{0}^{2} B_{1}^{2}}{\left(l-l^{\prime}\right)^{2}} \\
& \leqslant C(d) \lambda^{2} B_{1}^{-2}(2 q)^{2 m} \leqslant C(d) \lambda^{2}(2 q)^{2 m} .
\end{aligned}
$$

Следовательно, применяя неравенство (2.12) с $\beta+1=p q^{m}$, получаем оценку

$$
F\left(q^{m+1} p, l_{m}^{\prime}\right) \leqslant\left(C(d) \lambda^{2} q+1\right)^{2 m /\left(p q^{m}\right)} F\left(q^{m} p, l_{m}\right) .
$$

Итак, имеем

$$
F\left(q^{m} p, r_{m}\right) \leqslant\left(C(d) \lambda^{2}(q+1)\right)^{2 p^{-1} \Theta} F\left(p, \frac{1}{2}\right), \quad \Theta:=\Theta(d):=\sum_{m=1}^{\infty} m q^{-m} .
$$

Пусть $\left|S\left(\frac{1}{2}\right)\right|$ обозначает $(d+1)$-мерный объем $S\left(\frac{1}{2}\right)$. По неравенству Гёльдера

$$
F\left(p, \frac{1}{2}\right) \leqslant\left|S\left(\frac{1}{2}\right)\right|^{1 / p-1 / p_{0}} F\left(p_{0}, \frac{1}{2}\right) .
$$

Значит, для некоторого числа $c(d)$, зависящего только от $d$, получаем

$$
F\left(+\infty, \frac{1}{3}\right) \leqslant C_{4} F\left(p_{0}, \frac{1}{2}\right), \quad C_{4}=\left(3 \cdot 2^{d+1}\right)^{B_{1}^{2} / \lambda}=\exp \left\{c(d) B_{1}^{2}\right\} .
$$

2. Пусть $Q(l)=R(l) \times\left(1-l^{2}, 1\right)$, если $\frac{1}{3} \leqslant l \leqslant \frac{1}{2}$. При $p<0$ положим

$$
F(p, l)=\left(\iint_{Q(l)} u_{\varepsilon}^{p} d x d t\right)^{1 / p} .
$$

Аналогично этапу 1 при $\beta+1<0$ имеем

$$
F(\beta+1, l) \leqslant\left(C_{3}+1\right)^{1 /|\beta+1|} F\left(q(\beta+1), l^{\prime}\right) .
$$

Повторив предыдущее рассуждение, получаем

$$
F\left(-p_{0}, \frac{1}{2}\right) \leqslant C_{5} F\left(-\infty, \frac{1}{3}\right), \quad C_{5}=\exp \left\{c(d) B_{1}^{2}\right\} .
$$


3. Заметим, что $S\left(\frac{1}{2}\right) \subset D^{-}$и $Q\left(\frac{1}{2}\right) \subset D^{+}$. Согласно следствию 2.2 , имеем

$$
F\left(p_{0}, \frac{1}{2}\right) \leqslant C_{6} F\left(-p_{0}, \frac{1}{2}\right), \quad C_{6}=\exp \left\{c(d) B_{1}^{2}\right\} .
$$

На основании установленных неравенств получаем требуемую оценку для $u_{\varepsilon}$ вместо $u$ с постоянной, не зависящей от $\varepsilon$. Полагая $\varepsilon \rightarrow 0$, приходим к такой же оценке для $u$. Общий случай, когда $r \neq 1$ и $(\bar{x}, \bar{t}) \neq(0,1)$, сводится к рассмотренному случаю заменой переменных $(x, t) \rightarrow\left((x-\bar{x}) / r, 1+(t-\bar{t}) / r^{2}\right)$. Теорема доказана.

Наш следующий результат уточняет полученную оценку по отношению к зависимости от $r$. Его преимущество по сравнению с предыдущей теоремой состоит в том; что теперь $B^{2}$ входит в оценку без множителя $r$.

Теорема 2.5. Пусть $U\left(z_{0}, \theta r\right) \subset \Omega$ для некоторьих иисел $\theta>1 u$ $r>0$. Тогда при $0<s<t<1 u x, y \in U\left(z_{0}, r\right)$ для непрерьвной версии u выполнено следующее неравенство:

$$
u(y, s) \leqslant u(x, t) \exp \left\{K\left(\frac{|x-y|^{2}}{t-s}+(B+1)^{2} \frac{t-s}{\delta^{2}}+1\right)\right\}
$$

где $\delta=\min \{(\theta-1) r, \sqrt{s}\}$, а число $K$ зависит только от $d, \alpha$ u $\gamma$ следующим образом:

$$
K:=c(d)\left|1+\alpha^{-1}+\left(\alpha^{-1}+\alpha^{-1 / 2}\right) \gamma\right|^{2},
$$

причем $c(d)$ зависит только от $d$.

Д о к а за т ел ь с т в о. Зафиксируем $x \in U\left(z_{0}, r\right)$ и $y \in U\left(z_{0}, r\right)$. Пусть

$$
q_{0}=\frac{\delta}{9 d(B+1)} .
$$

Тогда $\delta<1, q_{0} B \leqslant 1$ и при $z \in U\left(z_{0}, r\right)$ имеем $R\left(z, 3 q_{0}\right) \subset U\left(z_{0}, \theta r\right) \subset \Omega$. Действительно, для всякого $z_{1} \in R\left(z, 3 q_{0}\right)$ имеем

$$
\left|z_{1}-z_{0}\right| \leqslant\left|z_{1}-z\right|+\left|z-z_{0}\right| \leqslant 3 d q_{0}+r \leqslant \theta r .
$$

Заметим, что при $s<\tau<t$ выполнено неравенство $\tau-9 q_{0}^{2} \geqslant s-$ $9 q_{0}^{2} \geqslant 0$, т.е. $\left(\tau-9 q_{0}^{2}, \tau\right) \subset[0,1]$. Следовательно, $Q\left(3 q_{0}\right)=R\left(z, 3 q_{0}\right) \times$ $\left(\tau-\Im q_{0}^{2}, \tau\right) \subset Q$.

Пусть $x_{n}=y+n(x-y) / N$ и $t_{n}=s+n(t-s) / N$, где $n=0,1,2, \ldots, N$. Тогда $y=x_{0}, s=t_{0}, x=x_{N}, t=t_{N}$ и

$$
\left|x_{n}-x_{n-1}\right|=\frac{|x-y|}{N}, \quad\left|t_{n}-t_{n-1}\right|=\frac{t-s}{N} .
$$

Положим

$$
q=\frac{1}{2}\left(\frac{1}{q_{0}^{2}}+\frac{56}{t-s}+\frac{256|x-y|^{2}}{(t-s)^{2}}\right)^{-1 / 2} .
$$


Тогда

$$
q \leqslant \min \left\{q_{0}, \sqrt{\frac{t-s}{56}}, \frac{t-s}{16|x-y|}\right\}
$$

Пусть

$$
N=\left[\frac{t-s}{8 q^{2}}\right]+1
$$

где $[r]$ обозначает целую часть $r$. Заметим, что при таком выборе $N$ выполняются следующие неравенства:

$$
\frac{2|x-y|}{q} \leqslant \frac{t-s}{8 q^{2}} \leqslant N, \quad \frac{t-s}{7 q^{2}}-\frac{t-s}{8 q^{2}}=\frac{t-s}{56 q^{2}} \geqslant 1 .
$$

Отсюда сразу получаем

$$
\left|x_{n}-x_{n-1}\right|=\frac{|x-y|}{N} \leqslant \frac{q}{2} \leqslant \frac{q_{0}}{2} .
$$

Так как $N$ - наименьшее натуральное число, большее $(t-s) /\left(8 q^{2}\right)$, и

$$
\frac{t-s}{7 q^{2}}-\frac{t-s}{8 q^{2}} \geqslant 1, \quad \text { то } \quad \frac{t-s}{8 q^{2}} \leqslant N \leqslant \frac{t-s}{7 q^{2}} .
$$

Следовательно,

$$
7 q^{2} \leqslant \frac{t-s}{N} \leqslant 8 q^{2}
$$

Учитывая, что $x_{n-1} \in R\left(x_{n}, q\right), 7 q^{2} \leqslant t_{n}-t_{n-1} \leqslant 8 q^{2}$ и

$$
R\left(x_{n}, 3 q\right) \times\left(t_{n}-9 q^{2}, t_{n}\right) \subset R\left(x_{n}, 3 q_{0}\right) \times\left(t_{n}-9 q_{0}^{2}, t_{n}\right) \subset Q,
$$

применим теорему $2.4 \mathrm{c} r=q$ и получим

$$
u\left(x_{n-1}, t_{n-1}\right) \leqslant \sup _{(z, \tau) \in Q^{*}(q)} u(z, \tau) \leqslant C_{0} \inf _{(z, \tau) \in Q(q)} u(z, \tau) \leqslant C_{0} u\left(x_{n}, t_{n}\right),
$$

где

$$
\begin{gathered}
Q^{*}(q)=R\left(x_{n}, q\right) \times\left(t_{n}-8 q^{2}, t_{n}-7 q^{2}\right), \quad Q(q)=R\left(x_{n}, q\right) \times\left(t_{n}-q^{2}, t_{n}\right), \\
C_{0}:=\exp \left(c(d)\left|1+\alpha^{-1}+\left(\alpha^{-1}+\alpha^{-1 / 2}\right) \gamma\right|^{2}\right) .
\end{gathered}
$$

В самом деле, мы имеем $q B \leqslant q_{0} B \leqslant 1$. Получаем следующее рекуррентное соотношение:

$$
u\left(x_{n-1}, t_{n-1}\right) \leqslant C_{0} u\left(x_{n}, t_{n}\right), \quad 0 \leqslant n \leqslant N .
$$

Следовательно, справедливо неравенство

$$
u(y, s)=u\left(x_{0}, t_{0}\right) \leqslant C_{0}^{N} u\left(x_{N}, t_{N}\right)=C_{0}^{N} u(x, t) .
$$

Подставляя указанное выше значение $N$, получаем

$$
u(x, s) \leqslant u(y, t) \exp \left\{K_{0}\left(\frac{t-s}{8 q^{2}}+1\right)\right\}
$$


где $K_{0}=\ln C_{0}$. Окончательно приходим к оценке

$$
u(y, s) \leqslant u(x, t) \exp \left\{K\left(\frac{|x-y|^{2}}{t-s}+(B+1)^{2} \frac{t-s}{\delta^{2}}+1\right)\right\}
$$

где $K=K(d, \alpha, \gamma)$ имеет нужный вид и $\delta=\min \{(\theta-1) r, \sqrt{s}\}$. Теорема доказана.

3 а м е ч а н и е 2.3. В случае, когда наш параболический оператор записан в недивергентной форме $L=\partial_{t}+a^{i j} \partial_{x_{i}} \partial_{x_{j}}+b^{i} \partial_{x_{i}}$, причем отображения $x \mapsto A(x, t)$ локально липшицевы равномерно по $t$, такая же нижняя оценка справедлива, если заменить $b^{i}$ на $b^{i}-\partial_{x_{j}} a^{i j}$.

3. Нижние оценки плотностей. Здесь мы получим поточечные оценки снизу для плотностей мер, удовлетворяющих слабым эллиптическим и параболическим уравнениям. Для этого будут использованы полученные выше результаты о постоянных в неравенстве Харнака. Дополнительно в эллиптическом случае мы получим достаточные условия для включения $\nabla \ln \varrho \in L^{p}(\mu)$ для всех $p>1$, что усиливает аналогичные результаты, полученные в [12].

Интересно сравнить полученные здесь нижние оценки с верхними оценками из работ [10] и [11], которые мы напомним для удобства читателя. Предположим, что вероятностная мера $\mu$ с плотностью $\varrho$ удовлетворяет уравнению (1.1) в смысле (1.2), т.е. в случае (I), причем отображения $A$ и $A^{-1}$ равномерно ограничены, $a^{i j}=a^{j i}$ равномерно липшицевы и при некотором $p>d$ имеет место включение $|b| \in L^{p}(\mu)$ (тогда $\mu$ удовлетворяет также уравнению (1.3)). Пусть дана положительная функция $\Phi \in W_{\mathrm{loc}}^{1,1}\left(\mathbf{R}^{d}\right)$. Тогда для выполнения оценки

$$
\varrho(x) \leqslant C \Phi(x)^{-1}
$$

достаточны следующие условия: $\Phi,|\nabla \Phi|^{p} \in L^{1}(\mu)$ (см. [11, теорема 3.1]). Например, если отображение $A$ равномерно ограничено, равномерно обратимо и равномерно липшицево, то для оценки

$$
\varrho(x) \leqslant C \exp \left(-\kappa|x|^{\beta}\right)
$$

с некоторыми $C>0, \beta>0$ и $\kappa>0$ достаточно следующих условий:

$$
\exp \left(M|x|^{\beta}\right) \in L^{1}(\mu), \quad|b(x)| \leqslant C_{0}+C_{1} \exp \left(M_{0}|x|^{\beta}\right), \quad 0 \leqslant M_{0}<d^{-1} M .
$$

Из всех перечисленных условий лишь одно - включение $\exp \left(M|x|^{\beta}\right) \in$ $L^{1}(\mu)$ - не выражено явно через $A$ и $b$. Чтобы обеспечить и это условие в терминах коэффициентов, достаточно иметь следующую оценку:

$$
(b(x), x) \leqslant c_{0}-c_{1}|x|^{\beta}, \quad c_{1}>M \beta \sup _{x}\|A(x)\| .
$$

Это вытекает из рассмотрения функции Ляпунова $V(x)=\exp \left(M|x|^{\beta}\right)$ при $|x| \geqslant 1$ (ср. [3], [11]). Поскольку $(A(x) x, x) \leqslant|x|^{2} \sup _{y}\|A(y)\|$, вне 
единичного шара имеем

$$
\begin{aligned}
L V(x)= & M \beta V(x)\left[M \beta|x|^{2 \beta-4}(A(x) x, x)+|x|^{\beta-2} \operatorname{Trace} A(x)\right. \\
& \left.\quad+(\beta-2)|x|^{\beta-4}(A(x) x, x)+|x|^{\beta-2}(b(x), x)\right] \\
\leqslant & c_{2}-c_{3}|x|^{\beta-2} V(x)
\end{aligned}
$$

при некоторых $c_{2}, c_{3}>0$. Отсюда следует, что функция $(|x|+1)^{\beta-2} V(x)$ интегрируема относительно $\mu$. Если $\beta \geqslant 2$, то получаем $V \in L^{1}(\mu)$; если $\beta<2$, то делаем то же самое с $\exp \left(M^{\prime}|x|^{\beta}\right)$, где $M^{\prime}>M$ таково, что $c_{1}>M^{\prime} \beta \sup _{x}\|A(x)\|$. Тогда $\mu$-интегрируемость $(|x|+1)^{\beta-2} \exp \left(M^{\prime}|x|^{\beta}\right)$ дает $\mu$-интегрируемость $\exp \left(M|x|^{\beta}\right)$.

Мы получим похожие, хотя и не в точности такого же порядка, оценки снизу; скажем, вместо $\kappa$ в неравенстве (3.1) будет некоторая другая постоянная в оценке снизу (но показатель $\beta$ не изменится).

В следующей теореме мы предполагаем, что неотрицательная локально ограниченная мера $\mu$ на $\mathbf{R}^{d}$ обладает плотностью $\varrho$, причем $\varrho \in W^{2,1}(U)$ для всякого шара $U \subset \mathbf{R}^{d}$. Пусть мера $\mu$ удовлетворяет уравнению (1.1) на $\mathbf{R}^{d}$ в смысле (1.3), т.е. в случае (II), где

$$
\mathscr{L}=\partial_{x_{i}}\left(a^{i j} \partial_{x_{j}}\right)+b^{i} \partial_{x_{i}}
$$

матричнозначное отображение $A=\left(a^{i j}\right)_{1 \leqslant i, j \leqslant d}$ измеримо, функции $\|A(x)\|$ и $\left\|A(x)^{-1}\right\|$ локально ограничены, $a^{i j}=a^{j i}$, а коэффициент $b=\left(b^{i}\right)_{i \leqslant d}$ - измеримое локально ограниченное векторное поле. Напомним, что, согласно доказанному в [6], в случае, когда $a^{i j} \in W_{\mathrm{loc}}^{p, 1}\left(\mathbf{R}^{d}\right)$ и $b^{i} \in L_{\mathrm{loc}}^{p}\left(\mathbf{R}^{d}\right)$ с некоторым $p>d$ и $A(x)^{-1}$ локально ограничено, всякое решение (1.1) в смысле (1.2) автоматически имеет плотность в $W_{\text {loc }}^{p, 1}\left(\mathbf{R}^{d}\right)$, поэтому удовлетворяет также (1.3).

Пусть $V$ - непрерывная возрастающая функция на $[0, \infty)$ и $V(0)>0$.

Теорема 3.1. Пусть $|b(x)| \leqslant V(|x| / \theta)$, где $\theta>1$, u пусть $\alpha(r):=$ $\sup _{|x| \leqslant r}\left\|A(x)^{-1}\right\|, \gamma(r):=\sup _{|x| \leqslant r}\|A(x)\|$. Tогда существует такое положительное число $K=K(d)$, зависящее только от $d$, что непрерьвная версия функции е удовлетворяет неравенству

$$
\varrho(x) \geqslant \varrho(0) \exp \left\{-K(d)(\theta-1)^{-1} \alpha(\theta|x|)^{-1}(\gamma(\theta|x|)+V(|x|)|x|)\right\} .
$$

$B$ частности, если $\|A(x)\| \leqslant \gamma u\left\|A(x)^{-1}\right\| \leqslant \alpha$, то существует такое положительное число $K=K(d, \alpha, \gamma, \theta)$, что непрерьвная версия

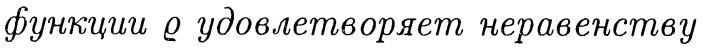

$$
\varrho(x) \geqslant \varrho(0) \exp \{-K(1+V(|x|)|x|)\} .
$$

Д оказат ельство. Зафиксируем $x$ и рассмотрим шар $U(0, \theta|x|)$. Теорема 2.2 дает нужную оценку, ибо $\sup _{z \in U(0, \theta|x|)}|b(z)| \leqslant$ $V(|x|)$. 
П р и м е р 3.1. Предположим, что условие (2.2) выполнено с $\Omega=\mathbf{R}^{d}$. Если для некоторых чисел $c_{1}, c_{2}>0$ при почти всех $x$ выполнена оценка

$$
|b(x)| \leqslant c_{1}|x|^{\beta}+c_{2}
$$

то существует такая постоянная $K$, что справедливо неравенство

$$
\varrho(x) \geqslant \varrho(0) \exp \left\{-K\left(1+|x|^{\beta+1}\right)\right\} .
$$

Если

$$
\begin{gathered}
\sup _{x, i, j}\left[\|A(x)\|+\left\|A(x)^{-1}\right\|+\left|\nabla a^{i j}(x)\right|\right]<\infty, \\
|b(x)| \leqslant c_{1}|x|^{\beta}+c_{2}, \quad \limsup _{|x| \rightarrow \infty}|x|^{-\beta-1}(b(x), x)<0,
\end{gathered}
$$

то на основании доказанного получаем двусторонние оценки

$$
\exp \left\{-K_{1}\left(1+|x|^{\beta+1}\right)\right\} \leqslant \varrho(x) \leqslant \exp \left\{-K_{2}\left(1+|x|^{\beta+1}\right)\right\} .
$$

Верхняя оценка следует из (3.1), если выбрать $M>0$ в (3.2), (3.3) достаточно малым. Например, если $A=\operatorname{Id}$ и $b^{i}(x)=x_{i}$, то мера с плотностью $\varrho(x)=\exp \left(-|x|^{2} / 2\right)$ является решением. Полученные выше результаты дают оценку

$$
\exp \left(-K_{1}\left(1+|x|^{2}\right)\right) \leqslant \varrho(x) \leqslant \exp \left(-K_{2}\left(1+|x|^{2}\right)\right)
$$

с некоторыми числами $K_{1}, K_{2}>0$, что достаточно правильно отражает характер убывания на бесконечности, хотя и не дает точной асимптотики.

Следует отметить, что условие $\lim \sup _{|x| \rightarrow \infty}|x|^{-\beta-1}(b(x), x)<0$ нужно лишь для того, чтобы обеспечить интегрируемость $\exp \left(M|x|^{\beta}\right)$, и может быть заменено последней.

$\mathrm{C}$ помощью полученных оценок можно указать эффективно проверяемые условия принадлежности к $L^{p}(\mu)$ логарифмического градиента $\nabla \varrho / \varrho$ меры $\mu$. В случае $p=2$ простые достаточные условия получены в работах [1], [2]. Первый общий результат для $p>2$ недавно установлен в работе [12]. Найденное ниже условие усиливает этот результат, поскольку мы не требуем дифференцируемости коэффициента сноса и используем меньшую регулярность коэффициента диффузии (в работе [12] предполагается, что $a^{i j} \in C^{3}\left(\mathbf{R}^{d}\right)$ и $b \in C^{2}\left(\mathbf{R}^{d}\right)$ ). Ослабление условий на коэффициенты стало возможным из-за того, что здесь, в отличие от [12], не используются методы теории нелинейных уравнений. Пусть $L^{p}(\mu)$ - пространство всех измеримых функций, которые интегрируемы в степени $p$ относительно меры $\mu$ с непрерывной положительной плотностью на $\mathbf{R}^{d}$, и пусть $W^{p, 1}(\mu)$ - соболевское пространство функций, которые принадлежат пространству $L^{p}(\mu)$ вместе с их обобщенными частными производными первого порядка. 
В следующей теореме и ее следствиях мы предположим, что $\mu-$ вероятностная мера на $\mathbf{R}^{d}$, удовлетворяющая эллиптическому уравнению (1.1) в смысле (1.2), т.е. мы имеем дело со случаем (I), $a^{i j}=a^{j i}$.

Tеорема 3.2. Пусть $a^{i j} \in C^{0, \delta}\left(\mathbf{R}^{d}\right) \cap W_{\text {loc }}^{p_{0}, 1}\left(\mathbf{R}^{d}\right), \quad \alpha \mathrm{Id} \leqslant A \leqslant \gamma \mathrm{Id}$, где $p_{0}>d u \alpha, \gamma, \delta>0$, причем $\lim _{r \rightarrow 0} \sup _{x}\left\|\partial_{x_{i}} a^{i j}\right\|_{L^{d}(U(x, r))}=0$ (nоследнее выполнено, если $A$ липшичево). Пусть положительная функция $\Phi \in W_{\text {loc }}^{1,1}\left(\mathbf{R}^{1}\right)$ возрастает на $[0,+\infty)$, причем $\Phi(N+1) \leqslant C \Phi(N)^{1+\varepsilon}$ при некоторых $C, \varepsilon>0$ и функиии $\Phi(|x|) u \Phi^{\prime}(|x|)^{p_{1}} c$ некоторьм $p_{1}>d$ интегрируемь по мере $\mu$ на $\mathbf{R}^{d}$. Предположим также, что даньи числа $C_{0}>0, \theta>1, p>1 u \gamma \in[0,1 / d)$, для которьх

$$
\begin{gathered}
|b(x)| \leqslant C_{0} \Phi(|x|-\theta)^{\gamma}, \quad\left|\nabla a^{i j}(x)\right|^{d} \leqslant C_{0} \Phi(|x|), \\
\sum_{N=1}^{\infty} N^{d-1} \Phi(N)^{-q}<\infty, \quad \text { zдe } \quad q:=1-\gamma(p+\varepsilon d)>0 .
\end{gathered}
$$

Тогда $\mu$ имеет плотность $\varrho$, для которой $\ln \varrho \in W^{p, 1}(\mu)$.

Д ок а з а т е л с с в о. Можно считать, что $\theta \leqslant 3$. Согласно [6], при указанных условиях $\mu$ имеет положительную непрерывную плотность $\varrho \in W_{\text {loc }}^{p_{0}, 1}\left(\mathbf{R}^{d}\right)$. По теореме 3.1 работы [11] при наших предположениях, обеспечивающих все условия этой теоремы, плотность $\varrho$ оценивается следующим образом:

$$
\varrho(x) \leqslant C_{1} \Phi(|x|)^{-1} .
$$

Так как ряд из $N^{d-1} \Phi(N)^{-q}$ сходится, то $\Phi(|x|)^{-q} \in L^{1}\left(\mathbf{R}^{d}\right)$, откуда $\varrho^{q} \in$ $L^{1}\left(\mathbf{R}^{d}\right)$ и $\ln \varrho \in L^{p}(\mu)$, ибо $0<q<1$ и $\varrho$ ограничена. При фиксированном $x \in \mathbf{R}^{d}$ имеем

$$
B:=\sup _{z \in U(x, \theta)}|b(z)| \leqslant C_{0} \sup _{z \in U(x, \theta)} \Phi(|x|-\theta)^{\gamma} \leqslant C_{0} \Phi(|x|)^{\gamma} .
$$

Пусть $0<r<\min \{1 / B, 1\}$. Тогда $r B<1$. Поэтому по теореме 2.2 сушествует такая постоянная $K=K(\alpha, \gamma, d, \theta, \delta)$, что для всякого $y \in$ $U\left(x, \theta^{1 / 2} r\right)$ выполнены неравенства

$$
K^{-1} \varrho(x) \leqslant \varrho(y) \leqslant K \varrho(x) .
$$

Оценим следующий интеграл:

$$
I:=\int_{U(x, r)} \frac{|\nabla \varrho(y)|^{p}}{\varrho(y)^{p-1}} d y .
$$

Согласно неравенству (3.5), имеем

$$
I \leqslant \varrho(x)^{1-p} K^{p-1} \int_{U(x, r)}|\nabla \varrho(y)|^{p} d y .
$$

Кроме того, справедлива оценка

$$
\int_{U\left(x, \theta^{1 / 2} r\right)}|b|^{p} \varrho^{p} d y \leqslant B^{p} \int_{U\left(x, \theta^{1 / 2} r\right)} \varrho^{p} d y .
$$


При $p>d$ следствие 2.1 с $h^{i}=b^{i} \varrho$ и неравенство (3.5) дают оценку

$$
\begin{aligned}
\int_{U(x, r)}|\nabla \varrho(y)|^{p} d y & \leqslant C_{2} r^{-p} \int_{U\left(x, \theta^{1 / 2} r\right)}\left[1+|b(y)|^{p}\right] \varrho(y)^{p} d y \\
& \leqslant C_{2} r^{-p}\left(1+r^{p} B^{p}\right)\|\varrho\|_{L^{p}\left(U\left(x, \theta^{1 / 2} r\right)\right)}^{p} \\
& \leqslant C_{2}^{\prime} r^{d-p}\left(1+r^{p} B^{p}\right) K^{p} \varrho(x)^{p},
\end{aligned}
$$

где числа $C_{2}$ и $C_{2}^{\prime}$ не зависят от $x$ и $r$. Такая же оценка верна при $p \leqslant d$. В самом деле, так как она верна для $p_{0}>d$, то неравенство Гёльдера и элементарно проверяемое неравенство $\left(1+t^{p_{0}}\right)^{p} \leqslant\left(1+t^{p}\right)^{p_{0}}$ дают

$$
\begin{aligned}
\|\nabla \varrho\|_{L^{p}(U(x, r))}^{p} & \leqslant\|\nabla \varrho\|_{L^{p_{0}}(U(x, r))}^{p / p_{0}} C(d) r^{d\left(p_{0}-p\right) / p_{0}} \\
& \leqslant C_{3} r^{\left(d-p_{0}\right) p / p_{0}} r^{d\left(p_{0}-p\right) / p_{0}}\left(1+r^{p} B^{p}\right) \varrho(x)^{p} \\
& =C_{3} r^{d-p}\left(1+r^{p} B^{p}\right) \varrho(x)^{p}
\end{aligned}
$$

где число $C_{3}$ не зависит от $r$ и $x$. Следовательно, из (3.6) находим

$$
I \leqslant C_{4} r^{d-p}\left(1+r^{p} B^{p}\right) \varrho(x) \leqslant 2 C_{4} r^{d-p} \varrho(x),
$$

где число $C_{4}$ не зависит от $x$ и $r$. Возьмем $r=\left(2 C_{0}\right)^{-1} \Phi(|x|)^{-\gamma}$, что возможно благодаря указанной выше оценке на $B$. Ввиду приведенной в начале доказательства оценки для $\varrho$, выполнено неравенство

$$
\int_{U(x, r)} \frac{|\nabla \varrho(y)|^{p}}{\varrho(y)^{p-1}} d y \leqslant C_{5} \Phi(|x|)^{\gamma p-\gamma d-1},
$$

где $C_{5}$ не зависит от $x$ и $r$. Положим $Q_{N}=U(0, N+1) \backslash U(0, N)$, $N>1$. Индукцией по $d$ легко показать, что есть такая постоянная $C_{6}$, зависящая лишь от $d$, что $Q_{N}$ можно покрыть шарами $U(x, r)$ радиуса $r=\left(2 C_{0}\right)^{-1} \Phi(N+1)^{-\gamma}$, общее число которых не превосходит $C_{6} C_{0}^{d} N^{d-1} \Phi(N+1)^{d \gamma}$. Из доказанного выше получаем

$$
I_{N}:=\int_{Q_{N}} \frac{|\nabla \varrho(y)|^{p}}{\varrho(y)^{p-1}} d y \leqslant C_{5} C_{6} C_{0}^{d} N^{d-1} \Phi(N+1)^{d \gamma} \Phi(N)^{\gamma p-\gamma d-1} .
$$

Поскольку $\Phi(N+1) \leqslant C \Phi(N)^{1+\varepsilon}$, то справедливо неравенство

$$
N^{d-1} \Phi(N+1)^{d \gamma} \Phi(N)^{\gamma p-\gamma d-1} \leqslant C N^{d-1} \Phi^{-q} .
$$

Значит, $\sum_{N=1}^{\infty} I_{N}<\infty$. Следовательно, $|\nabla \ln \varrho|^{p} \varrho \in L^{1}\left(\mathbf{R}^{d}\right)$. Теорема доказана.

Следствие 3.1. Пусть $a^{i j} \in C^{0, \delta}\left(\mathbf{R}^{d}\right) \cap W_{\text {loc }}^{p_{0}, 1}\left(\mathbf{R}^{d}\right), \alpha \operatorname{Id} \leqslant A \leqslant \gamma \mathrm{Id}$, где $\alpha, \gamma, \delta>0$ и $p_{0}>d>1$, причем $\lim _{r \rightarrow 0} \sup _{x}\left\|\partial_{x_{i}} a^{i j}\right\|_{L^{d}(U(x, r))}=0$. Пусть $p>1$. Предположим, что при некоторых $M>0$ и $\beta>0$ бункиия $\exp \left(M|x|^{\beta}\right)$ интегрируема по мере $\mu$ на $\mathbf{R}^{d}$, причем

$$
|b(x)| \leqslant C_{0} \exp \left\{\kappa|x|^{\beta}\right\}, \quad\left|\nabla a^{i j}(x)\right| \leqslant C_{0} \exp \left\{\kappa|x|^{\beta}\right\},
$$


где $0<\kappa d \max (p, d)<M$. Тогда $\ln \varrho \in W^{p, 1}(\mu)$. В частности, если для всякого $\kappa>0$ найдется такое число $C(\kappa)$, что

$$
|b(x)|+\left|\nabla a^{i j}(x)\right| \leqslant C(\kappa) \exp \left\{\kappa|x|^{\beta}\right\},
$$

$m o \ln \varrho \in W^{p, 1}(\mu)$ для всех $p \in[1,+\infty)$.

Д о к а з а т е л ь с т в о. Найдется такое достаточно малое число $\varepsilon_{0}>0$, что для $\gamma:=\max (p, d)^{-1}-\varepsilon_{0}$ получим $\gamma>0$ и $\kappa \gamma^{-1}<M d^{-1}$. Возьмем в качестве $\Phi$ функцию $\Phi(r)=\exp \left(M_{\theta}|r|^{\beta}\right)$, где $M_{0}$ выбрано в $\left(\kappa \gamma^{-1}, M d^{-1}\right)$. Поскольку $p \gamma<1$, то найдется такое $\varepsilon>0$, что $q=$ $1-\gamma(p+\varepsilon d)>0$. Положим $\theta=2$. Заметим, что

$$
\exp \left(\kappa r^{\beta}\right) \leqslant C_{1} \exp \left(\gamma M_{0}|r-2|^{\beta}\right),
$$

ибо $\kappa<\gamma M_{0}$. Поэтому $|b(x)| \leqslant C_{0} C_{1} \Phi(|x|-2)^{\gamma}$. Ясно также, что при некотором $p_{1}>d$ функция $\Phi^{\prime}(|x|)^{p_{1}}$ интегрируема относительно $\mu$, ибо $M_{0} d<M$. Как легко видеть, выполнены и остальные условия теоремы 3.2. Следствие доказано.

Аналогично доказывается следующее утверждение (берем $\Phi(r)=$ $\left.r^{\beta}+1\right)$.

Следствие 3.2. Пусть $a^{i j} \in C^{0, \delta}\left(\mathbf{R}^{d}\right) \cap W_{\text {loc }}^{p_{0}, 1}\left(\mathbf{R}^{d}\right), \alpha \operatorname{Id} \leqslant A \leqslant \gamma \mathrm{Id}$, где $\alpha, \gamma, \delta>0$ и $p_{0}>d>1$, причем $\lim _{r \rightarrow 0} \sup _{x}\left\|\partial_{x_{i}} a^{i j}\right\|_{L^{d}(U(x, r))}=0$. Пусть $p>1$. Предположим, что при некотором $\beta>d$ бункиия $|x|^{\beta d}$ интегрируема по мере $\mu$ на $\mathbf{R}^{d}$, причем

$$
|b(x)| \leqslant C_{0}+C_{0}|x|^{\beta \gamma}, \quad\left|\nabla a^{i j}(x)\right| \leqslant C_{0}+C_{0}|x|^{\beta / d},
$$

где $0<\gamma<d^{-1}, \gamma<1-d \beta^{-1}$. Тогда $\ln \varrho \in W^{p, 1}(\mu)$ при $p \in[1,(\beta-$ d) $\left.\beta^{-1} \gamma^{-1}\right)$.

Перейдем к параболическому уравнению. Как и в эллиптическом случае, напомним некоторые верхние оценки. Пусть вероятностная мера $\mu$ на $\mathbf{R}^{d} \times[0,1)$ удовлетворяет параболическому уравнению (1.1) в смысле (1.4) с начальным условием $\mu_{0}=\varrho(\cdot, 0) d x$ (см. (1.6)), причем отображения $A$ и $A^{-1}$ равномерно ограничены, отображения $x \mapsto A(x, t)$ равномерно липшицевы с некоторой общей постоянной, $a^{i j}=a^{j i},|b| \in$ $L^{\beta}(\mu)$ при некотором $\beta>d+2$ и $\sup _{t \in(0,1)}\|b(\cdot, t)\|_{L^{d}\left(\mu_{t}\right)}<\infty$. Предположим, что дана такая функция $\Phi \geqslant c>0$ на $\mathbf{R}^{d}$ с локально ограниченными производными второго порядка, что $\varrho(x, 0) \leqslant C \Phi(x)^{-1}, \Phi \in L^{1}\left(\mu_{0}\right)$ и

$$
\begin{gathered}
\Phi^{1+\varepsilon}, \quad|L \Phi|^{\beta / 2} \Phi^{1-\beta / 2}, \quad|A \nabla \Phi|^{\beta} \Phi^{1-\beta} \in L^{1}(\mu), \\
\sup _{t \in[0,1)} \int_{\mathbf{R}^{d}} \Phi(x) \varrho(x, t) d x<\infty
\end{gathered}
$$

с некоторым $\varepsilon>0$. Тогда, согласно теореме 3.3 работы [10], которую можно использовать, перейдя к недивергентной форме нашего уравнения, для всякого $\tau<1$ найдется такое число $C_{\tau}$, что

$$
\varrho(x, t) \leqslant C_{\tau} \Phi(x)^{-1} \quad \text { для почти всех }(x, t) \in \mathbf{R}^{d} \times[0, \tau] .
$$


Например, пусть $A$ и $A^{-1}$ равномерно ограничены, $A$ равномерно липшицево по $x$, причем функция $\exp \left(K|x|^{r}\right) \varrho(x, 0)$ ограничена и интегрируема по $\mathbf{R}^{d}$ для некоторого $r>0$. Предположим также, что

$$
\begin{gathered}
|b| \in L^{\beta}(\mu), \quad \exp \left((2 K+\varepsilon)|x|^{r}\right) \in L^{1}(\mu), \\
\sup _{t \in[0,1)} \int_{\mathbf{R}^{d}} \exp \left(K|x|^{r}\right) \varrho(x, t) d x<\infty, \sup _{t \in(0,1)}\|b(\cdot, t)\|_{L^{d}\left(\mu_{t}\right)}<\infty
\end{gathered}
$$

для некоторых $\beta>d+2$ и $\varepsilon>0$. Тогда для всякого $\tau<1$ найдется такое число $C(\tau)>0$, что

$$
\varrho(x, t) \leqslant C(\tau) \exp \left(-K|x|^{r}\right), \quad(x, t) \in \mathbf{R}^{d} \times[0, \tau] .
$$

Чтобы обеспечить (3.7) в терминах $A$ и $b$, достаточно иметь интегрируемость $\exp \left(K^{\prime}|x|^{r}\right) \varrho(x, 0)$ с некоторым $K^{\prime}>\max \left(2 K, C_{2}(d+2)\right)$ и следующие оценки:

$$
\sup _{t}|b(x, t)| \leqslant C_{1} \exp \left(C_{2}|x|^{r}\right) \quad \text { и } \quad \sup _{t}(b(x, t), x) \leqslant C_{3}-C_{4}|x|^{r}
$$

для некоторых $C_{1}, C_{2}, C_{3}, C_{4}>0 \mathrm{c}$

$$
2 K r \sup _{t, x}\|A(x, t)\|<C_{4} \quad \text { и } \quad C_{2}(d+2) r \sup _{t, x}\|A(x, t)\|<C_{4} .
$$

Это проверяется с помощью функции Ляпунова $V$ такой, что $V(x)=$ $\exp \left(M|x|^{r}\right)$ при $|x| \geqslant 1$ и $M$ выбрано так, что $\max \left(2 K, C_{2}(d+2)\right)<$ $M<K^{\prime}, M r \sup _{t, x}\|A(x, t)\|<C_{4}$. Из (3.4) следует, что функция $L V$ ограничена сверху некоторой постоянной $K_{0}$, что согласно лемме 2.2 в [22] дает оценку

$$
\int_{\mathbf{R}^{d}} V(x) \mu_{t}(d x) \leqslant K_{0} t+\int_{\mathbf{R}^{d}} V(x) \varrho(x, 0) d x .
$$

Как мы сейчас увидим, имеются похожие нижние оценки; однако различие между верхними и нижними оценками более значительно, чем в эллиптическом случае.

Пусть измеримое матричнозначное отображение $A=\left(a^{i j}\right)_{1 \leqslant i, j \leqslant d}$ на $\mathbf{R}^{d} \times(0,1)$ удовлетворяет условию $(2.10)$ с $\Omega=\mathbf{R}^{d}, a^{i j}=a^{j i}$, и пусть $b-$ измеримое векторное поле на множестве $\mathbf{R}^{d} \times(0,1)$.

Пусть $V$ - непрерывная возрастающая функция на $[0, \infty)$ и $V(0)>0$.

Теорема 3.3. Пусть $\sup _{t \in(0,1)}|b(x, t)| \leqslant V(|x| / \theta)$ для почти всех $x \in \mathbf{R}^{d}$, где $\theta>1$. Пусть

$$
\alpha(r):=\sup _{t \in(0,1),|x| \leqslant r}\left\|A(x, t)^{-1}\right\|, \quad \gamma(r):=\sup _{t \in(0,1),|x| \leqslant r}\|A(x, t)\| .
$$

Предположим, что $\mu$ - неотрицательная мера с плотностью $\varrho н а$ $\mathbf{R}^{d} \times(0,1)$ такой, что

$$
\varrho \in \mathbf{H}^{2,1}(U \times J)
$$


для всякого шара $U \subset \mathbf{R}^{d}$ и всякого отрезка $J$ в $(0,1)$. Предположим, что н удовлетворяет уравнению (1.1) в смысле (1:5), т.е. в случае (II). Тогда существует такое положительное число $K=K(d)$, что непрерывная версия функиии @ удовлетворяет неравенству

$$
\begin{aligned}
\varrho(x, t) \geqslant \varrho(0, s) \exp & \left\{-K(d) \mid 1+\alpha(\theta|x|)^{-1}+\left(\alpha(\theta|x|)^{-1} .\right.\right. \\
& \left.\left.+\alpha(\theta|x|)^{-1 / 2}\right)\left.\gamma(\theta|x|)\right|^{2}\left(1+\frac{t-s}{s} V(|x|)^{2}+\frac{1}{t-s}|x|^{2}\right)\right\},
\end{aligned}
$$

где $0<s<t<1, x \in \mathbf{R}^{d}$. В иастности, если $\|A(x, t)\| \leqslant \gamma u$ $\left\|A(x, t)^{-1}\right\| \leqslant \alpha$, то существует такое положительное число $K=$ $K(d, \alpha, \gamma, \theta)$, что непрерывная версия функиии $\varrho$ удовлетворяет неравенству

$$
\varrho(x, t) \geqslant \varrho(0, s) \exp \left\{-K\left(1+\frac{t-s}{s} V(|x|)^{2}+\frac{1}{t-s}|x|^{2}\right)\right\}
$$

где $0<s<t<1, x \in \mathbf{R}^{d}$.

Доказательство. В теореме 2.5 возьмем $\Omega=U(0, \theta|x|)$ и $y=0$. Это дает искомую оценку.

Следствие 3.3. Если в ситуации теоремы 3.3 при почти всех $t \in(0,1)$ функиия $x \mapsto \varrho(x, t)$ не равна тождественно нулю, то для всякого отрезка $\left[\tau_{1}, \tau_{2}\right]$ в $(0,1)$ существует такое иисло $K=$ $K\left(d, \alpha, \gamma, \theta, \tau_{1}, \tau_{2}\right) \geqslant 0$, что при всех $t \in\left[\tau_{1}, \tau_{2}\right]$ u $x \in \mathbf{R}^{d}$ выполнены неравенства

$$
\exp \left(-K\left(1+V(|x|)^{2}+|x|^{2}\right)\right) \leqslant \varrho(x, t) \leqslant \exp \left(K\left(1+V(|x|)^{2}+|x|^{2}\right)\right) .
$$

Д о к а 3 а т е л ь с т в о. Из теоремы 2.5 и нашего условия следует, что функция $t \mapsto \varrho(0, t)$ не обращается в нуль на интервале $(0,1)$. Рассмотрим отрезок $\left[\tau^{\prime}, \tau^{\prime \prime}\right]$, где $\tau^{\prime}=\tau_{1} / 2, \tau^{\prime \prime}=1-\left(1-\tau_{2}\right) / 2$. Первая из доказываемых нами оценок следует из теоремы 3.3 , в которой следует взять $s=\tau^{\prime}$. Вторая оценка ясна из теоремы 2.5 .

П р и м е $\mathrm{p} 3.2$. Пусть в ситуации теоремы 3.3 матрицы $A(x, t)$ и $A(x, t)^{-1}$ равномерно ограничены и для некоторых постоянных $c_{1}>0$ и $c_{2}>0$ при почти всех $x$ выполнено неравенство

$$
\sup _{t \in(0,1)}|b(x, t)| \leqslant c_{1}|x|^{\beta}+c_{2} .
$$

Тогда существует такое положительное число $K$, что

$$
\varrho(x, t) \geqslant \varrho(0, s) \exp \left\{-K\left(1+\frac{t-s}{s}|x|^{2 \beta}+\frac{1}{t-s}|x|^{2}\right)\right\} .
$$

Например, если $L=\partial_{t}+\frac{1}{2} \Delta$, то мера $(2 \pi t)^{-1 / 2} e^{-|x|^{2} /(2 t)} d x d t$ является решением. Наши результаты дают такое число $K>0$, что $\varrho \geqslant e^{-K(\delta)|x|^{2} / t}$ 
в любой полосе $\mathbf{R}^{d} \times(\delta, 1)$, где $\delta>0$. Аналогичным образом наша нижняя оценка точна в случае линейного коэффициента сноса, но в случае квадратичного роста $|b|$ она становится уже менее точной; например, если $\varrho(x, t)=C \exp \left(-|x|^{3}\right)$, то в нашей нижней оценке появляется $\exp \left(-K|x|^{4}\right)$.

Укажем·набор условий на коэффициенты $A$ и $b$, обеспечивающих двусторонние экспоненциальные оценки плотности решения в параболическом случае.

П р и м е р 3.3. Предположим, что отображения $A$ и $A^{-1}$ равномерно ограничены, функции $x \mapsto a^{i j}(x, t)$ равномерно липшицевы с общей постоянной, $a^{i j}=a^{j i}$, причем для некоторых $r>1, \sigma \geqslant 0, K>0$, $K^{\prime}>K$ имеем

$$
\begin{array}{cl}
|b(x, t)| \leqslant C+C|x|^{r-1+\sigma}, & \varrho(x, 0) \leqslant C \exp \left(-K^{\prime}|x|^{r}\right), \\
(x, b(x, t)) \leqslant c_{1}-c_{2}|x|^{r}, & c_{2}>2 r K \sup _{x, t}\|A(x, t)\| .
\end{array}
$$

Предположим, что вероятностная мера $\mu$ на $\mathbf{R}^{d} \times(0,1)$ удовлетворяет уравнению (1.1) в смысле (1.4), т.е. в случае (I). Тогда $\mu$ обладает такой непрерывной плотностью $\varrho$, что для всякого отрезка $\left[\tau_{1}, \tau_{2}\right] \subset(0,1)$ найдутся такие числа $C_{1}, C_{2}$ и $K_{0}$, что

$$
C_{1} \exp \left(-K_{0}|x|^{2 r+2 \sigma-2}-K_{0}|x|^{2}\right) \leqslant \varrho(x, t) \leqslant C_{2} \exp \left(-K|x|^{r}\right),
$$

где $(x, t) \in \mathbf{R}^{d} \times\left[\tau_{1}, \tau_{2}\right]$. Верхняя оценка следует из примера 3.1 работы [10], а нижняя из доказанного выше. В отличие от эллиптического случая здесь не совпадают показатели степени при $|x|$ в нижней и верхней оценках. Отметим, что указанные условия дают также и существование решения $\mu=\mu_{t} d t$ с вероятностными мерами $\mu_{t}$ при произвольном начальном распределении (см. [8], [22]). Проблема единственности рассмотрена в [23].

Еще одно применение наших результатов связано с доказательством существования конечной энтропии решения по пространственной переменной при положительных $t$ для всякого начального распределения. Наличие конечной энтропии, полезное во многих отношениях, необходимо для применения результатов из [10], дающих интегрируемость $|\nabla \varrho(x, t)|^{2} / \varrho(x, t)$.

Пусть $W$ - непрерывная возрастающая функция на полупрямой $[0, \infty), W(0)>0$ и $\lim _{r \rightarrow \infty} W(r)=+\infty$.

Предложение 3.1. Пусть матриць $A(x, t)$ u $A(x, t)^{-1}$ равномерно ограничень, $a^{i j}=a^{j i}$ и при некотором $\theta>1$ выполнено следуюшее неравенство:

$$
\sup _{t \in(0,1)}|b(x, t)|^{2} \leqslant W\left(\frac{|x|}{\theta}\right), \quad x \in \mathbf{R}^{d} .
$$


Пусть $\mu$ - мера вида $\mu=\mu_{t} d t$, где каждая $\mu_{t}-$ вероятностная мера, причем $\mu$ удовлетворяет условию (3.8) и уравнению (1.1) в смьсле (1.5). Предположим, что

$$
\int_{0}^{1} \int_{\mathbf{R}^{d}}\left[|x|^{2}+W(|x|)\right] \mu_{t}(d x) d t<\infty .
$$

Тогда для всякого отрезка $\left[\tau_{1}, \tau_{2}\right] \subset(0,1)$ имеем

$$
\int_{\tau_{1}}^{\tau_{2}} \int_{\mathbf{R}^{d}} \varrho(x, s)|\ln \varrho(x, s)| d x d s<\infty .
$$

Доказ ат ель с т в. Пусть отрезок $\left[\tau_{1}, \tau_{2}\right] \subset(0,1)$ фиксирован. Согласно следствию 3.3 , существует такое число $K=$ $K\left(d, \alpha, \gamma, \theta, \tau_{1}, \tau_{2}\right)>0$, что

$$
\exp \left\{-K\left(1+|x|^{2}+W(|x|)\right)\right\} \leqslant \varrho(x, s) \leqslant \exp \left\{K\left(1+|x|^{2}+W(|x|)\right)\right\},
$$

где $s \in\left[\tau_{1}, \tau_{2}\right], x \in \mathbf{R}^{d}$. По теореме Фубини при почти каждом $s \in(0,1)$ функция $x \mapsto\left(|x|^{2}+W(|x|)\right) \varrho(x, s)$ интегрируема по $\mathbf{R}^{d}$. Для таких $s$ из $\left[\tau_{1}, \tau_{2}\right]$ получаем

$$
\int_{\mathbf{R}^{d}} \varrho(x, s)|\ln \varrho(x, s)| d x \leqslant \int_{\mathbf{R}^{d}} K\left(1+|x|^{2}+W(|x|)\right) \varrho(x, s) d x .
$$

Тем самым, предложение доказано.

Следствие 3.4. Предположим, что $A \in H_{\mathrm{loc}}^{2,1}\left(\mathbf{R}^{d} \times(0,1)\right), a^{i j}=a^{j i}$, причем $A(x, t)$ и $A(x, t)^{-1}$ равномерно ограничень. Кроме того, предположим, что при некотором $\theta>1$ выполнено следующее неравенство:

$$
\sup _{t \in(0,1)}|b(x, t)|^{2} \leqslant W\left(\frac{|x|}{\theta}\right), \quad x \in \mathbf{R}^{d} .
$$

Пусть $\mu$ - мера вида $\mu=\mu_{t} d t$, где каждая $\mu_{t}$ - вероятностная мера, причем выполнено условие (3.8). Мы предполагаем, что н удовлетворяет уравнению (1.1) в смысле (1.5) и имеет такое начальное условие $\mu_{0}$, что функиия $W_{0}(x):=W(|x|)$ интегрируема относительно $\mu_{0}$. Наконеи, пусть

$$
W(r) \geqslant c_{1}+c_{2} r^{2} \quad \text { u } \quad \mathscr{L} W_{0}(x) \leqslant C
$$

с некоторыми постоянньми $c_{1}, c_{2}, C>0$. Тогда для непрерьвной версии உлотности $\mu$ имеем

$$
\sup _{s \in(0,1)} \int_{\mathbf{R}^{d}} \varrho(x, s)|\ln \varrho(x, s)| d x<\infty .
$$

Доказательство. Положим $b_{0}^{i}:=\partial_{x_{j}} a^{i j}+b^{i}$. Тогда $\mathscr{L}=$ $\partial_{t}+a^{i j} \partial_{x_{i}} \partial_{x_{j}}+b_{0}^{i} \partial_{x_{i}}$. Следовательно, мы можем применять результаты работы [22], в которой рассматривались операторы в недивергентной форме. Согласно лемме 2.2 из [22], для почти всех $s \in[0,1)$ имеем

$$
\int_{\mathbf{R}^{d}} W(|x|) \varrho(x, s) d x \leqslant C s+\int_{\mathbf{R}^{d}} W(|x|) \mu_{0}(d x) .
$$


Ввиду непрерывности $\varrho$ и теоремы Фату это неравенство верно для всех $s \in(0,1)$, что позволяет применить то же рассуждение, что и в предыдущем предложении.

Подчеркнем еще раз, что если функции $x \mapsto a^{i j}(x, t)$ локально липшицевы равномерно по $t \in(0,1)$, то в теореме 3.3 , следствии 3.3 , примере 3.2 , предложении 3.1 и следствии 3.4 всякое решение $\mu$ уравнения (1.1) в смысле (1.4) удовлетворяет условию (3.8), следовательно, является решением и в смысле (1.5).

П р и м е р 3.4. Предположим, что в следствии 3.4 дополнительно известно, что функции $x \mapsto a^{i j}(x, t)$ равномерно липшицевы с общей постоянной. Тогда для всякого отрезка $\left[\tau_{1}, \tau_{2}\right] \subset(0,1)$ ввиду теоремы 2.1 работы [10] имеем

$$
\int_{\tau_{1}}^{\tau_{2}} \int_{\mathbf{R}^{d}} \frac{|\nabla \varrho(x, t)|^{2}}{\varrho(x, t)} d x d t<\infty .
$$

$\Pi$ р и м е $\mathrm{p}$ 3.5. Предположим, что отображения $A(x, t)$ и $A(x, t)^{-1}$ равномерно ограничены, функции $x \mapsto a^{i j}(x, t)$ равномерно липшицевы с общей постоянной и что

$$
|b(x, t)| \leqslant c_{0} \exp \left(c|x|^{r}\right), \quad(b(x, t), x) \leqslant c_{1}-c_{2}|x|^{r}, \quad c_{2}>2 c r \sup _{x, t}\|A(x, t)\| .
$$

Пусть $\mu$ - вероятностная мера на $\mathbf{R}^{d} \times(0,1)$, удовлетворяющая уравнению (1.1) в смысле (1.4) с таким начальным условием $\mu_{0}$, что функция $\exp \left(c|x|^{r}\right)$ интегрируема относительно $\mu_{0}$. Тогда

$$
\int_{\tau_{1}}^{\tau_{2}} \int_{\mathbf{R}^{d}} \frac{|\nabla \varrho(x, t)|^{2}}{\varrho(x, t)} d x d t<\infty
$$

для всякого отрезка $\left[\tau_{1}, \tau_{2}\right] \subset(0,1)$. Для обоснования этого примера достаточно взять $W_{0}(z)=\exp \left(M|z|^{r}\right)$ с $M>2 c$, достаточно близким к $2 c$.

\section{СПИСОК ЛИТЕРАТУРЫ}

1. Bogachev V.I., Röckner M. Regularity of invariant measures on finite- and infinitedimensional spaces and applications. - J. Funct. Anal., 1995, v. 133, № 1, p. 168-223.

2. Bogachev V.I., Krylov N.V., Röckner M. Regularity of invariant measures: the case of nonconstant diffusion part. - J. Funct. Anal., 1996, v. 138, № 1, p. 223-242.

3. Богачев В.И., Рёкнер М. Обобщение теоремы Хасьминского об инвариантных мерах. - Теория вероятн. и ее примен., 2000, т. 45, в. 3, с. 363-378.

4. Богачев В. И., Крылов Н. В., Рёкнер М. Дифференцируемость инвариантных мер и переходных вероятностей сингулярных диффузий. - Докл. РАН, 2001, т. 376, № 2, c. 151-154.

5. Bogachev V.I., Röckner M., Wang F.-Y. Elliptic equations for invariant measures on finite and infinite dimensional manifolds. - J. Math. Pures Appl., 2001, v. 80, №2, p. 177-221. 
6. Bogachev V.I., Krylov N. V., Röckner M. On regularity of transition probabilities and invariant measures of singular diffusions under minimal conditions. - Comm. Partial Differential Equations, 2001, v. 26, № 11-12, p. 2037-2080.

7. Богачев В. И., Рёкнер М., Штаннат В. Единственность решений эллиптических уравнений и единственность инвариантных мер диффузий. - Матем. сб., 2002, т. 193 , № 7 , c. 3-36.

8. Bogachev V.I., Da Prato G., Röckner M. Existence of solutions to weak parabolic equations for measures. - Proc. London Math. Soc., 2004, v. 88, № 3, p. 753-774.

9. Богачев В. И., Крылов Н.В., Рёкнер М. Регулярность и глобальные оценки плотностей инвариантных мер диффузионных процессов. - Докл. РАН, 2005, т. 405, № 5 , c. $583-587$.

10. Богачев В. И., Рёкнер М., Шапошников С. В. Глобальная регулярность и оценки решений параболических уравнений. - Теория вероятн. и ее примен., 2005, т. 50, в. 4 , c. $652-674$.

11. Bogachev V.I., Krylov N. V., Röckner M. Elliptic equations for measures: regularity and global bounds of densities. - J. Math. Pures Appl., 2006, v. 85, № 6, 743-757.

12. Metafune G., Pallara D., Rhandi A. Global regularity of invariant measures. J. Funct. Anal., 2005, v. 223, № 2, p. 396-424.

13. Morrey C. B. Multiple Integrals in the Calculus of Variations. New York: SpringerVerlag, 1966, $506 \mathrm{c.}$

14. Шапошников С.В. Оценка Морри соболевских норм решений эллиптических уравнений. - Матем. заметки, 2006, т. 79, № 3, с. 450-469.

15. Lunardi A., Vespri V. Hölder regularity in variational parabolic nonhomogeneous equations. - J. Differential Equations, 1991, v. 94, № 1, p. 1-40.

16. Byun S.-S. Elliptic equations with BMO coefficients in Lipschitz domains. - Trans. Amer. Math. Soc., 2005, v. 357, № 3, p. 1025-1046.

17. Krylov N. V. Parabolic and elliptic equations with VMO coefficients. - Comm. Partial Differential Equations, 2007, v. 32, № 3, p. 453-475.

18. Ладыженская O. А., Солонников B. А., Уральцева Н. Н. Линейные и квазилинейные уравнения параболического типа. М.: Наука, 1967, 736 с.

19. Гилбарг Д., Трудингер Н. Эллиптические дифференциальные уравнения с частными производными второго порядка. М.: Наука, 1989, 463 с.

20. Aronson D. G., Serrin J. Local behavior of solutions of quasilinear parabolic equations. - Arch. Rational Mech. Anal., 1967, v. 25, p. 81-122.

21. Moser J. A Harnack inequality for parabolic differential equations. - Comm. Pure Appl. Math., 1964, v. 17, p. 101-134; corrections: ibid., 1967, v. 20, p. 231-236.

22. Bogachev V.I., Da Prato G., Röckner M. On parabolic equations for measures. Preprint BiBoS № 06-05-213, Bielefeld: Bielefeld University, 2006; Comm. Partial Differential Equations (to appear).

23. Bogachev V. I., Da Prato D., Röckner M., Stannat W. Uniqueness of solutions to weak parabolic equations for measures. - Preprint BiBoS № 06-07-225, Bielefeld: Bielefeld University, 2006; Proc. London Math. Soc. (to appear).

Поступила в редакцию

1.XII.2006 